\title{
Measurements of Bunch Motion Due to the Longitudinal Dipole-Coupled Bunch Instability at the Cornell Electron-Positron Storage Ring
}

\author{
Robert Holtzapple \\ Stanford Linear Accelerator Center, Stanford University, Stanford CA 94309 \\ Laboratory of Nuclear Studies, Cornell University, Ithaca, NY 14853 \\ Michael Billing \\ Laboratory of Nuclear Studies, Cornell University, Ithaca, NY 14853
}

Published in Physical Review Special Topics-Accelerators and Beams

${ }^{+}$This work was supported by the National Science Foundation Work and by the Department of Energy contract DE-AC03$76 \mathrm{SF} 00515$. 


\title{
Measurements of Bunch Motion Due to the Longitudinal Dipole- Coupled Bunch Instability at the Cornell Electron-Positron Storage Ring
}

\author{
Robert Holtzapple $^{1,2}$ and Michael Billing ${ }^{1}$ \\ ${ }^{1}$ Laboratory of Nuclear Studies, Cornell University, Ithaca, NY 14853 \\ ${ }^{2}$ Stanford Linear Accelerator Center, Stanford University, Stanford, CA 94309
}

\begin{abstract}
In the past a longitudinal dipole-coupled bunch instability had limited high current operation at CESR and resulted in a degradation of luminosity performance. A longitudinal feedback system successfully damps this instability and the exchange of superconducting rf cavities for normal conducting rf cavities in CESR has further reduced the instability's strength. A description of the longitudinal dynamics with the instability present are described in this paper along with detailed measurements of the instability using a dual-axis synchroscan streak camera. The measurements were made on single trains of bunches, multiple trains, and colliding beams. These measurements give a characterization of the instability's degradation of luminosity, modes of oscillation, and bunch distribution changes.
\end{abstract}

\section{Introduction}

To achieve high luminosity, the Cornell Electron-Positron Storage Ring (CESR) is operated with multiple bunches. Under present conditions, there are up to nine trains of bunches, with up to five bunches in each train, for a maximum of 45 positron and electron bunches (Fig.1). One limitation to high luminosity is a longitudinal dipole-coupled bunch instability (LDCBI) which occurs when multiple bunches are present. The LDCBI was first noted during operation with nine trains of two bunches in CESR. At the instability threshold, a rapid growth of the spectral lines at the synchrotron sidebands of the rotation harmonics was observed. The growth of amplitude for the spectral lines depends upon the spacing of the trains and bunches and it is the indication for when the instability threshold is exceeded. The threshold of the LDCBI depends on the spacing between bunches and the number of bunches in each train. The LDCBI degrades the machine luminosity and, at high current, is responsible for beam loss.

To combat the LDCBI, two modifications were made: (i) a low Q, $1.1 \mathrm{GHz}$. accelerating cavity is used as a longitudinal feedback cavity to damp the LDCBI [1], and (ii) to increase the current threshold and reduce the effect of the LDCBI, four single cell niobium superconducting rf cavities have been installed in CESR replacing 20 cells of normal conducting $\mathrm{rf}$ cavities. The superconducting rf cavities have more strongly damped higher order modes than the previous normal conducting rf cavities [2]. Measurements of the LDCBI at CESR have been made using a dual sweep synchroscan streak camera. The change in the characteristics of the instability due to the superconducting rf cavities will be discussed. A previous characterization of the LDCBI was performed with a single sweep steak camera with the normal conducting rf cavities. A single sweep 
streak camera can measure only single bunch dynamics; therefore, detailed information of bunch oscillations has not been previously measured [3].

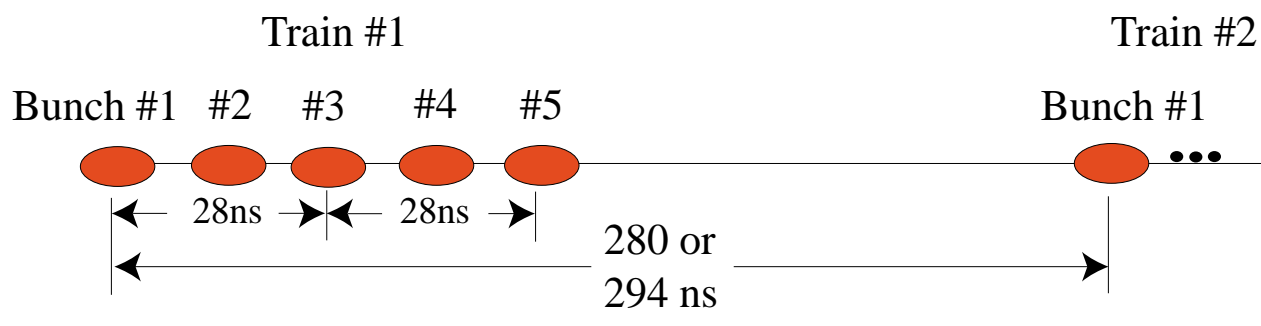

FIG. 1. (Color) There are nine trains of bunches in CESR. Each train is separated by 280 or $294 \mathrm{~ns}$ and can have up to five bunches per train. The spacing between bunches is $14 \mathrm{~ns}$.

\section{Dipole-Mode Longitudinal Coupled Bunch Instability and the Longitudinal Dynamics of CESR}

Several aspects of the longitudinal dipole-coupled bunch instability are presented in the section. The measurements made for this paper do not provide enough information to make a complete theoretical model of multiple bunch longitudinal dynamics in CESR, but, instead, several aspects of longitudinal dynamics observed in the presence of the LDCBI will be presented. A good starting place is the description of single particle longitudinal dynamics, followed by a description of multiple bunch dynamics that relate to the instability, such as time oscillation, bunch distributions, and luminosity degradation.

\section{(A) Single particle longitudinal dynamics at low current}

For a single particle, ignoring collective effects (low current), the longitudinal phase space for electrons, or positrons, in a circular accelerator exhibit motion described by the following differential equations [4]:

$$
\begin{aligned}
& \frac{\mathrm{d} \tau}{\mathrm{dt}}=-\alpha \delta, \\
& \frac{\mathrm{d} \delta}{\mathrm{dt}}=\frac{1}{\mathrm{E}_{0} \mathrm{~T}_{0}}\left[\mathrm{eV}(\tau)-\mathrm{U}_{\mathrm{rad}}(\delta)\right],
\end{aligned}
$$

where $\tau$ and $\delta$ are the temporal and fractional energy deviations of a particle with respect to the synchronous particle, where $\alpha$ is the momentum compaction factor, $\mathrm{T}_{0}$ is the circulation time, $\mathrm{E}_{0}$ is the beam energy, and $\operatorname{eV}(\tau)-\mathrm{U}_{\text {rad }}(\delta)$ is the net energy gain from the rf voltage $[\mathrm{eV}(\tau)]$ and synchrotron radiation losses $\left[\mathrm{U}_{\mathrm{rad}}(\delta)\right]$. From these equations and the assumption that the particles being described radiate very many small quanta during their circulation around the ring, the particles in the bunch will have a Gaussian distribution in both phase space coordinates, 


$$
\mathrm{f}_{0}(\tau, \delta)=\frac{1}{2 \pi \sigma_{\tau} \sigma_{\delta}} \exp \left(-\frac{\tau^{2}}{2 \sigma_{\tau}^{2}}-\frac{\delta^{2}}{2 \sigma_{\delta}^{2}}\right)
$$

where the standard deviations in both coordinates are related by the synchrotron oscillation angular frequency, $\omega_{s}$,

$$
\omega_{s}=\sqrt{\frac{\alpha \mathrm{e} \dot{\mathrm{V}}}{\mathrm{E}_{0} \mathrm{~T}_{0}}}, \quad \sigma_{\tau}=\frac{\sigma_{\delta}}{\omega_{s}}=\frac{\sigma_{\mathrm{z}}}{\mathrm{c}},
$$

where $\dot{\mathrm{V}}=\frac{\mathrm{dV}_{\mathrm{RF}}}{\mathrm{dt}}$. The standard deviation bunch length in a storage ring is

$$
\sigma_{\tau}=\frac{\alpha}{\omega_{s}} \sqrt{\mathrm{C}_{\mathrm{q}} \mathrm{E}_{0}^{2}\left(\frac{\mathrm{I}_{3}}{2 \mathrm{I}_{2}+\mathrm{I}_{4}}\right)},
$$

where $\mathrm{I}_{2}, \mathrm{I}_{3}$ and $\mathrm{I}_{4}$ are the synchrotron integrals, and $\mathrm{C}_{\mathrm{q}}$ is the constant of $3.84 \times 10^{-13} \mathrm{~m}$. The synchrotron integrals and longitudinal parameters are noted in Table I for the conditions under which the measurements were made.

\begin{tabular}{|c|c||c|c|}
\hline $\mathrm{I}_{1}$ & $8.53 \mathrm{~m}$ & $\sigma_{\mathrm{E}} / \mathrm{E}$ & $6.728 \times 10^{-4}$ \\
\hline $\mathrm{I}_{2}$ & $1.047 \times 10^{-1} \mathrm{~m}^{-1}$ & $\sigma_{\mathrm{Z}}$ & $1.719 \times 10^{-2} \mathrm{~m}$ \\
\hline $\mathrm{I}_{3}$ & $2.300 \times 10^{-3} \mathrm{~m}^{-2}$ & $\mathrm{E}_{0}$ & $5.289 \mathrm{GeV}$ \\
\hline $\mathrm{I}_{4}$ & $1.482 \times 10^{-3} \mathrm{~m}^{-1}$ & $\mathrm{~T}_{0}$ & $2.56 \times 10^{-6} \mathrm{sec}$ \\
\hline $\mathrm{v}_{\mathrm{rf}}$ & $500 \mathrm{MHz}$ & $\alpha$ & $1.11 \times 10^{-2}$ \\
\hline $\mathrm{U}_{0}$ & $1.14 \mathrm{MeV}$ & $\mathrm{V}_{\mathrm{rf}}$ & $6.00 \mathrm{MV}$ \\
\hline
\end{tabular}

TABLE I. The synchrotron integral and longitudinal parameters for CESR.

The longitudinal phase space $(\tau, \delta)$ may be described by Hamiltonian dynamics. The Hamiltonian is determined from Eqs. (1) and (2) where the net acceleration voltage function, $e V(\tau)-U_{\operatorname{rad}}(\delta)$, describes the change in the energy of the particles due to synchrotron radiation, the rf accelerating voltage, longitudinal wake potential, and any external time-dependent voltage $\mathrm{eV}_{\mathrm{ext}}$. The function is given by

$$
\mathrm{eV}(\tau)-\mathrm{U}_{\mathrm{rad}}(\delta)=\mathrm{eV}_{\mathrm{rf}} \sin \left(\omega_{\mathrm{rf}} \tau+\phi\right)-\mathrm{U}_{\mathrm{rad}}+\mathrm{eV}_{\mathrm{ext}}
$$

where $\mathrm{eV}_{\mathrm{rf}} \sin \left(\omega_{\mathrm{rf}} \tau+\varphi\right)$ is the $\mathrm{rf}$ accelerating voltage and $\mathrm{U}_{\mathrm{rad}}$ is the average energy lost due to synchrotron radiation. The Hamiltonian $\mathrm{H}=\mathrm{H}(\delta, \tau ; \mathrm{t})$ which describes the longitudinal motion of the bunch is given by

$$
\mathrm{H}(\delta, \tau, \mathrm{t})=\frac{\alpha^{2} \delta^{2}}{2}-\frac{\alpha}{\mathrm{T}_{0} \mathrm{E}_{0}}\left\{\left(\mathrm{U}_{\mathrm{rad}}-\mathrm{eV}_{\mathrm{ext}}\right) \tau+\frac{\mathrm{eV}_{\mathrm{rf}}}{\omega_{\mathrm{rf}}} \cos \left(\omega_{\mathrm{rf}} \tau+\varphi\right)\right\},
$$

and the phase potential, $\Phi(\tau)$ for the rf bucket is given by the second term in the expression or 


$$
\Phi(\tau)=\frac{-\alpha}{\mathrm{T}_{0} \mathrm{E}_{0}}\left(\left(\mathrm{U}_{\mathrm{rad}}-\mathrm{V}_{\mathrm{ext}}\right) \tau+\frac{\mathrm{eV}_{\mathrm{rf}}}{\omega_{\mathrm{rf}}} \cos \left(\omega_{\mathrm{rf}} \tau+\phi\right)\right) .
$$
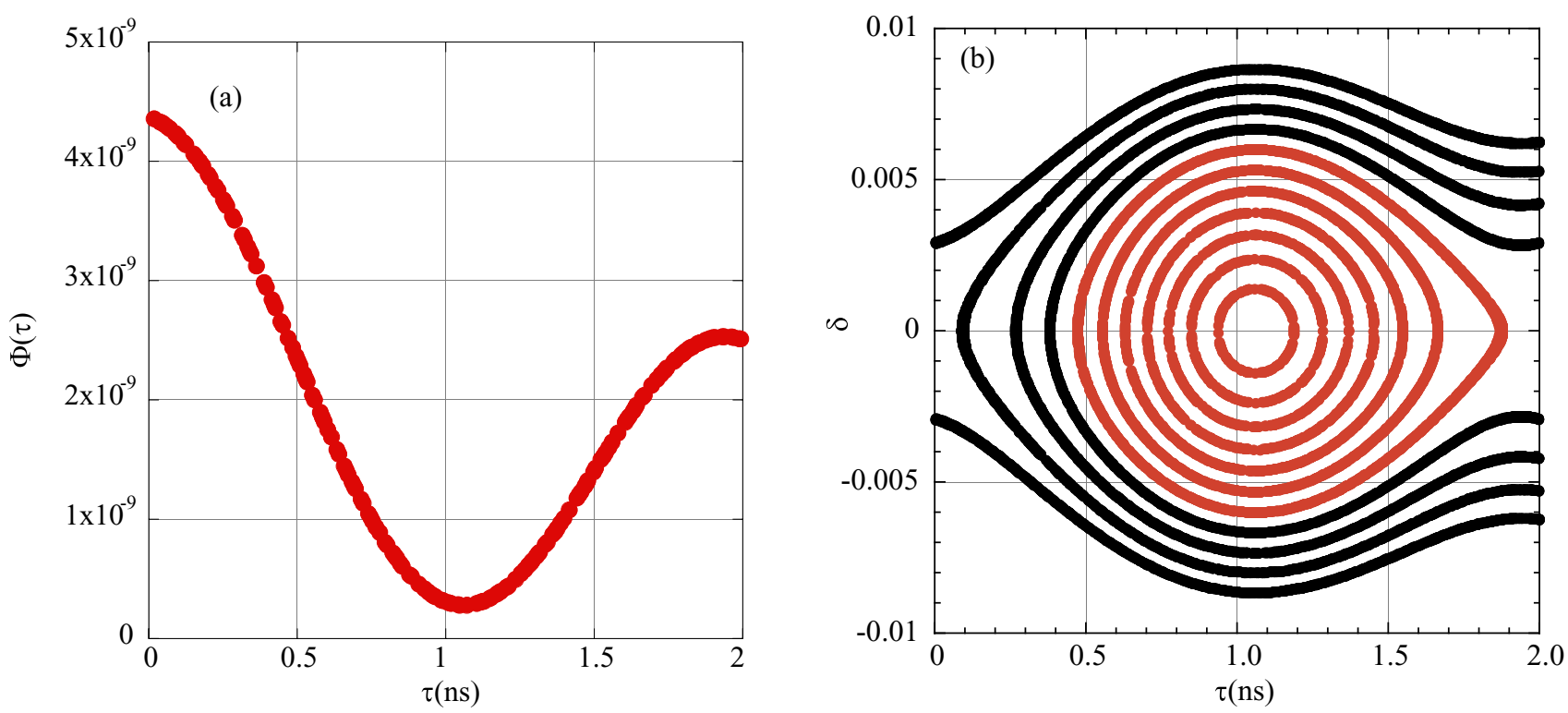

FIG. 2. (Color) (a) The normalized potential energy ignoring the external potential term and (b) the longitudinal phase space for the CESR rf bucket using the parameters in Table I.

To illustrate this for CESR, the shape of the rf bucket has been calculated by varying the $\tau$ and $\delta$ coordinates while keeping the Hamiltonian fixed. The potential energy and rf bucket are plotted in Fig. 2. Several features can be pointed out. (i) The asymmetry in the potential energy is due to the average synchrotron radiation loss. (ii) The red lines represent the stable phase space trajectories within the rf bucket plotted for particles having equal increments in fractional energy error. The separatrix is the phase space trajectory having the maximum amplitude for a captured particle. (iii) The black lines represent the trajectories of particles that will not be captured in the rf bucket. Ignoring radiation damping, a particle that is injected onto one of these trajectories will remain on that trajectory as it moves in phase space.

In a storage ring, which has synchrotron radiation losses dominated by the emission of a large number of small quanta, the equilibrium distribution is Gaussian in fractional energy deviation and temporal deviation as mentioned above. If one were to apply an external periodic voltage $\left(\mathrm{V}_{\text {ext }}\right)$ varying slowly in time, the phase space trajectories would become time modulated. Since this slowly varying modulating voltage can be viewed as adding to the rf system's average voltage, the centroid of the rf bucket will be modulated in time. If this modulation frequency is close to the synchrotron oscillation frequency, the displacement of the bunch's centroid from equilibrium can be driven to relatively large amplitudes in terms of the rf bucket. In Fig. 2, we see that, for particles with large amplitudes, since the phase potential $\Phi(\tau)$ is weaker than a parabolic potential at large amplitude (due to the cosine function), the phase space trajectories tend to stretch farther apart for large excursions in $\tau$. This stretching of phase space trajectories produces a modulation in the observed symmetry of the temporal and energy distribution of a bunch executing large amplitude oscillations. This asymmetry 
should be most visible at the largest excursions of the centroid in $\tau$. A pictorial representation, ignoring potential well distortion (single-bunch wakefield), of the dynamics in longitudinal phase space is given in Fig. 3. The asymmetric temporal distributions displayed in Fig. 3(b) are determined from Eq. (4). The convention of the asymmetric distribution is as follows: (i) when the asymmetry factor is negative the front (head) of the distribution is shorter and the back (tail) of the distribution is longer; (ii) a positive asymmetry factor has a longer head and shorter tail; (iii) when the asymmetry factor is zero, the distribution is a Gaussian distribution. The asymmetry factor will approximately be an odd function of the bunch's temporal displacement. The energy distribution, not pictured in Fig. 3, will be modulated in shape as well.
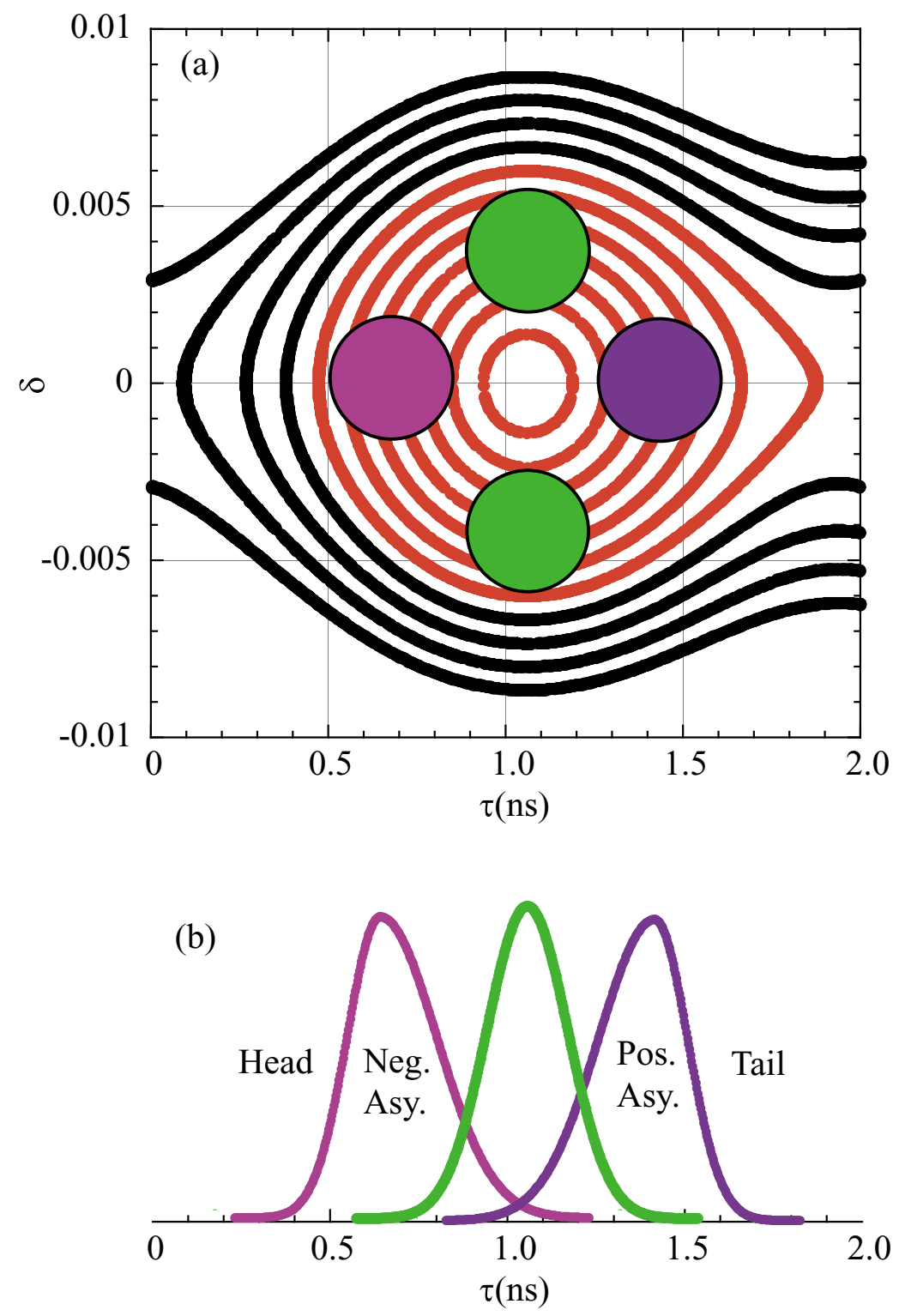

FIG. 3. (Color) (a) A bunch experiencing a large amplitude oscillation in longitudinal phase space and (b) a temporal projection of the bunch at three different displacements in longitudinal phase space. The temporal bunch distribution is symmetric or asymmetric depending on its position in longitudinal phase space. 


\section{(B) Multiple bunch longitudinal dynamics}

The spacing of multiple bunches in CESR is shown in Fig. 1. At low current (below the instability threshold) the longitudinal dynamics of all the bunches is similar to that of a single bunch. As the current increases the instability appears. The instability has been identified as a longitudinal dipole-coupled bunch instability, which is only observed with multiple bunches per train. The detailed characteristics of the instability are described elsewhere [5]. The threshold of the instability depends upon the bunch spacing. We observed that (i) at the instability threshold current for some value of $n$, a signal at $n f_{r} \pm f_{s}$ appears in the beams spectra, where $f_{r}$ is the rotation frequency and $f_{S}$ is the synchrotron frequency. The presence of this signal indicates predominately dipole motion in phase space; (ii) the threshold current for the instability is dependent on the bunch spacing in the train; (iii) the instability reduces the CESR high-energy physics luminosity; (iv) when the normal conducting rf cavities were present in CESR, as the current is increased well above the instability threshold a signal at $\mathrm{mf}_{\mathrm{r}} \pm 2 \mathrm{f}_{\mathrm{s}}$ appears, for some value of $\mathrm{m}$ [3]. This higher mode of the bunch's oscillation has not been observed with the superconducting cavities up to $350 \mathrm{~mA}$ in a single beam.

High intensity bunches are sources of electromagnetic fields called wakefields, which react back on the bunches in the storage ring. The wakefields are caused by discontinuities in the vacuum chamber walls and can, therefore, be modified by changing the shape of the vacuum chambers in the storage ring. Such a change has occurred with the installation of the superconducting rf cavities. The wakefields maybe modeled by the addition of a term $-V_{\text {Wake }}(\tau)$ to the net accelerating voltage term in the Hamiltonian, to describe the beam-induced voltage. The addition of wakefields to the description of the longitudinal dynamics can produce a number of effects, such as a change in the equilibrium bunch shape due to potential well distribution, the creation of unstable dipole motion of the bunches, and the distortion of the bunch's longitudinal distribution. Although a detailed theoretical description is outside of the scope of this paper, a qualitative description follows [6]. The dipole-mode corresponds to the rigid motion of the centroid of the bunch about its equilibrium point in longitudinal phase space. Figure 4 shows two possible unstable modes of oscillation. If the amplitude of the wakefield decays in a time which is long, in comparison to bunch spacing, adjacent bunches, and even adjacent trains, will affect each other. If the decay is rapid, then the bunches ignore each other.

The wakefields that couple from bunch to bunch maybe viewed as analogues to $V_{\text {ext }}$, described previously, which can drive the bunches to large amplitudes in their rf buckets. Consequently, we would expect large amplitude oscillations driven by the wakefields to change the bunch distribution, as shown as an example in Fig. 3(b). This change in bunch distribution has been measured in CESR as a change in the bunch asymmetry and is discussed later in this paper. Although in some instances the large amplitude oscillation can cause particle loss, generally in CESR the oscillation amplitude tends to limit due, presumably, to the nonlinearities of rf bucket's longitudinal restoring force. 
(a)

(b)

(c)

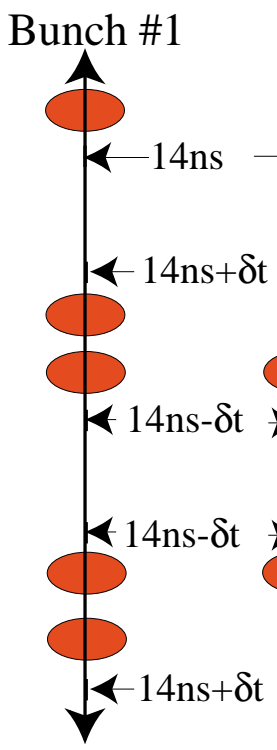

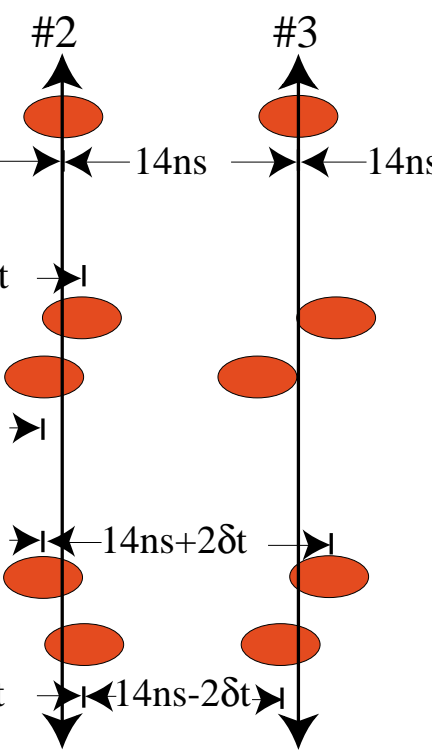

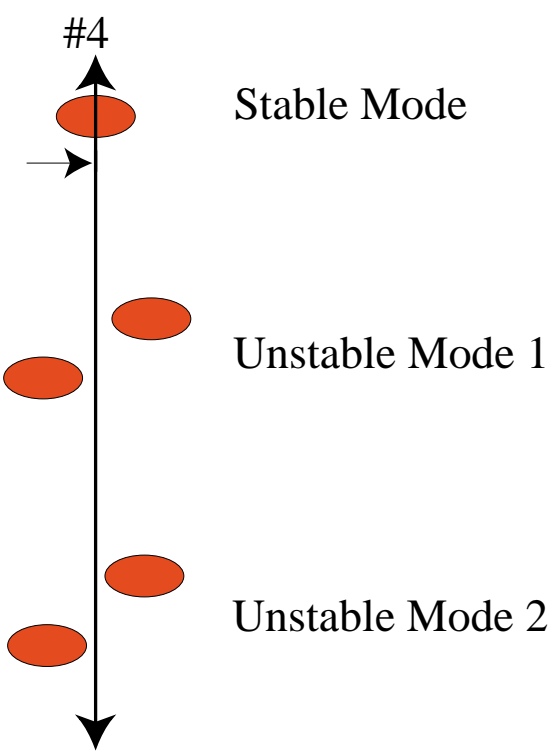

FIG. 4. (Color) A description of the dipole modes of oscillation is made with this diagram. If the bunches are stable and arrive at $14 \mathrm{~ns}$ intervals they are depicted in (a). Two possible unstable modes of oscillation are depicted in (b) and (c).

The rigid centroid motion of bunches due to the LDCBI has another consequence. The time oscillation of the bunches degrades the luminosity in CESR. The luminosity of an electron-positron storage ring is

$$
L \approx \frac{f_{0} N \gamma \xi}{2 r_{e} \beta_{V}^{*}},
$$

where $\mathrm{N}$ is the number of particles per bunch, $\mathrm{f}_{0}$ is the revolution frequency, $\mathrm{r}_{\mathrm{e}}$ is the classical electron radius, $\xi$ is the beam-beam tune shift parameter, $\gamma=E / m c^{2}$, and $\beta_{\mathrm{V}}^{*}$ is the vertical betatron function at the interaction point which happens to be $1.8 \mathrm{~cm}$ during this experiment. The luminosity is inversely proportional to the vertical betatron function so that a large amplitude LDCBI oscillation will cause the bunch's collision point to vary longitudinally and, thereby, interact on the average at a larger value of the betatron function. A reduction factor can be determined from the oscillation of the bunches at the interaction point. The betatron function at the interaction point varies as

$$
\beta_{\mathrm{v}}(\mathrm{s})=\beta_{\mathrm{v}}{ }^{*}+\frac{\mathrm{s}^{2}}{\beta_{\mathrm{v}}{ }^{*}},
$$

where $\mathrm{s}$ is the location from the center point of the interaction point. Milton calculated the effect of bunch length on luminosity (the "hourglass effect") for the present conditions, but with head-on collisions rather than angle crossing collisions [7]. He defines the suppression factor $\mathrm{S}_{\mathrm{f}}$, which is the unitless factor multiplying the short bunch length luminosity $\mathrm{L}_{0}$, as

$$
L=L_{0} S_{f}\left(\sigma_{z}, \beta_{v}^{*}\right),
$$

where 


$$
\mathrm{L}_{0}=\frac{\mathrm{N}^{2} \mathrm{f}_{0}}{4 \pi \sigma_{\mathrm{x}} \sigma_{\mathrm{y}}},
$$

and $\sigma_{x}, \sigma_{y}$ are the beam's transverse sizes at the interaction point. For the present case of $\beta_{\mathrm{v}}^{*}=\sigma_{\mathrm{z}}=18 \mathrm{~mm}$, results in $\mathrm{S}_{\mathrm{f}}=0.81$. The ratio of luminosity when the beams are unstable/stable gives the reduction factor of the luminosity as

$$
\frac{\mathrm{L}_{\mathrm{u}}}{\mathrm{L}_{\mathrm{s}}}=\frac{\mathrm{S}_{\mathrm{f}}\left(\sigma_{\mathrm{z}}\right)_{\mathrm{u}}}{\mathrm{S}_{\mathrm{f}}\left(\sigma_{\mathrm{z}}\right)_{\mathrm{s}}},
$$

where the subscript u's are unstable conditions and the subscript s's are stable conditions. A measurement of the degradation of luminosity is presented later in the paper.

\section{Streak Camera}

The characteristics of the longitudinal dipole-coupled bunch instability were measured using synchrotron light from either the electron or positron bunches and a dual-sweep synchroscan streak camera (DSSSC). The synchrotron light is emitted from the bunch as it travels through a dipole magnet. The light is extracted and transported from the vacuum chamber to the streak camera by a series of mirrors over a path of $17 \mathrm{~m}$.

To understand the operation of the DSSSC, Fig. 5 depicts the major components of the camera. The workings of the DSSSC can be described in the following manner. Consider four pulses of light that are separated in time as they enter the streak camera [Fig. 5(a)]. These four pulses can be tracked through the camera to illustrate its basic operations. The four pulses enter the streak camera through a pinhole slit that has, typically, a $50 \mu \mathrm{m}$ diameter. The pulses are transported through the streak camera input optics which form an image of the pinhole slit onto a photocathode. As the pulses hit the photocathode of the streak tube, they are converted into photoelectrons. The number of photoelectrons produced is proportional to the number of the photons in the input pulse. Here the photoelectron pulses are again spaced by their arrival time. The emitted photoelectron pulses are accelerated by an accelerating mesh, which pulls the photoelectrons away from the photocathode and keeps the pulses separated in time, and then they are deflected by two plates, a fast synchroscan sweep plate and a slow sweep deflection plate. The fast synchroscan plate is triggered by a $143 \mathrm{MHz}$ timing signal. The frequency of $143 \mathrm{MHz}$ was chosen to allow the bunches to be on every voltage sweep when the bunch spacing has an integral bunch spacing of $7 \mathrm{~ns}$. In routine operation of CESR, bunches are spaced in multiples of $14 \mathrm{~ns}$. The sweep for the fast plates oscillate and data are acquired on every voltage sweep near the zero crossing [Fig. 5(b)]. If the slow sweep is turned off, the fast sweep superimposes all bunches within the camera's exposure time. The synchroscan unit alone intensifies weak light pulses by repetitive sweeps over a long exposure time. The slow sweep, which is variable between $100 \mathrm{~ns}$ to $100 \mathrm{~ms}$, sweeps the other plane and separates the four pulses [Fig. 5(c)]. By sweeping both plates, simultaneous measurement of several pulses of light can occur [Fig. 6(a)]. 
After the two deflection plates, the electrons enter the microchannel plate which multiplies the electron pulse by several thousand times. Then the photoelectrons strike a phosphor screen where they are converted into light again. The brightness of the phosphor screen is proportional to the intensity of the pulse. The vertical axis on the phosphor screen is the fast time axis and the horizontal axis is the slow time axis [Fig. 6(a)]. The image on the phosphor is transported by the output optics to a cooled charge-coupled device camera where the image is then digitized and processed by a computer. Longitudinal profiles of an image are to be analyzed off-line.

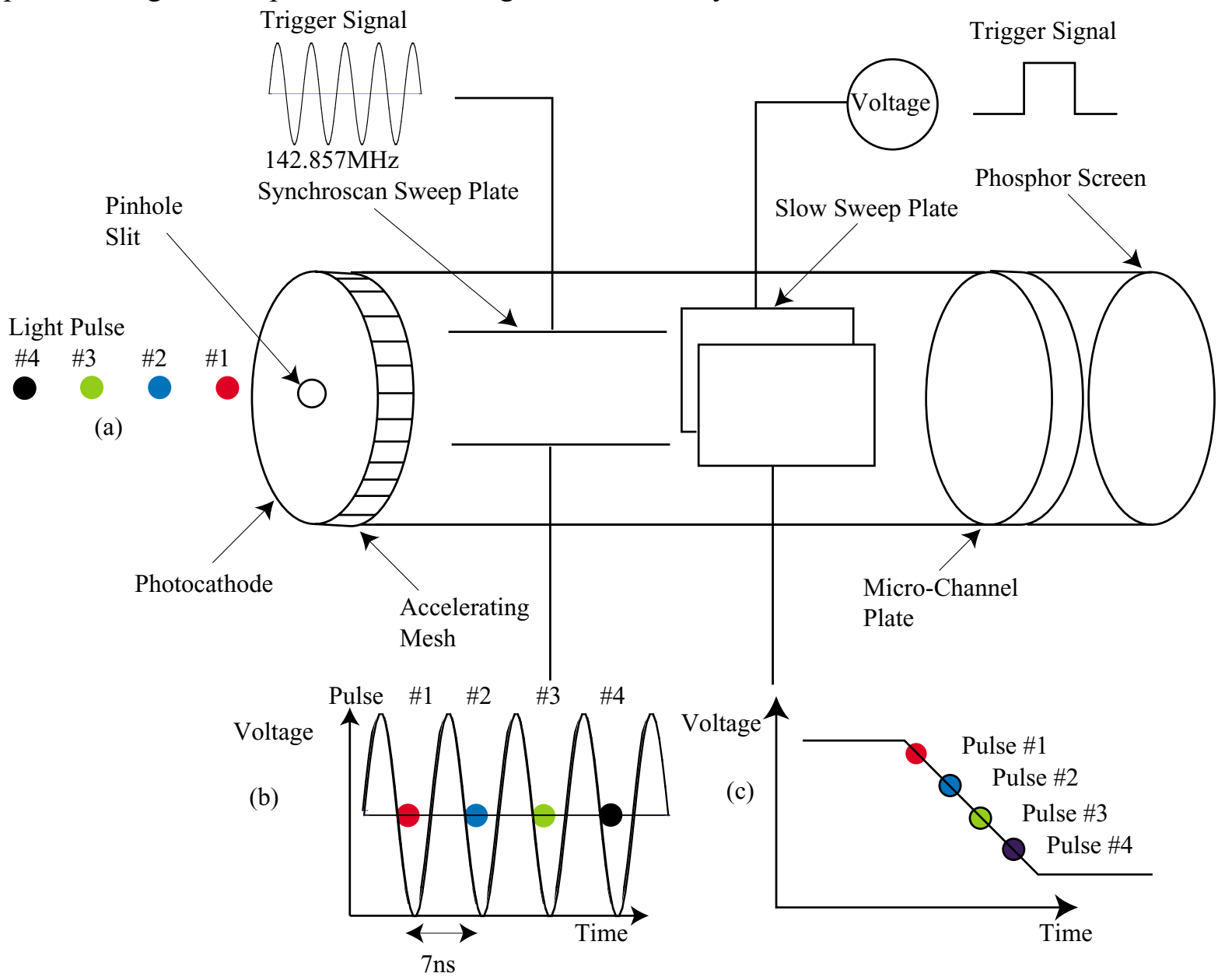

FIG. 5. (Color) (a) The four light pulses that enter the streak camera are separated in time, (b) the four sets of photoelectrons arriving on the zero crossing of the oscillating voltage, and (c) the four pulses arriving on the slow sweep voltage where each photoelectron is deflected at a different angle dependent on its arrival time.

A typical streak camera image is shown in Fig. 7. The longitudinal information of each light pulse (trains in this instance) is extracted in the following way. Each pulse is selected with the vertical cursors shown in the Fig. 7. Between the vertical cursors, the pixels are summed horizontally to create the longitudinal profile of the bunch distribution (displayed on the left-hand side of the image). For each image, a longitudinal profile for each light pulse is created, saved, and processed off-line. The processing includes fitting the longitudinal profile to an asymmetric Gaussian function given by 


$$
I(z)=I_{0}+I_{1} \exp \left\{-\frac{1}{2}\left(\frac{(z-\bar{z})}{(1+\operatorname{sgn}(z-\bar{z}) A) \sigma}\right)^{2}\right\},
$$

where $I_{0}=$ pedestal and $I_{1}=$ peak of the asymmetric Gaussian. The term $\operatorname{sgn}(z-\bar{z}) A$ is the asymmetry factor that parameterized the shape of the asymmetric Gaussian.
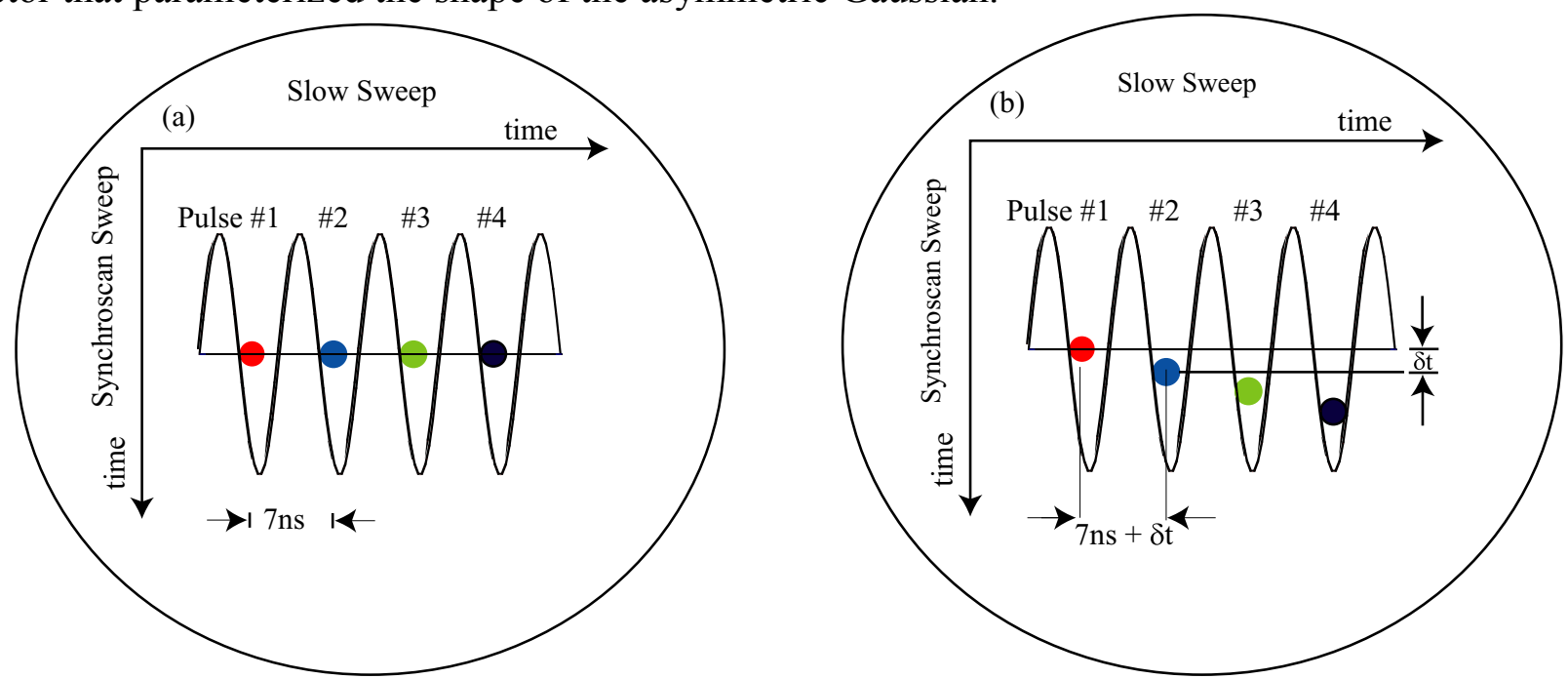

FIG. 6. (Color) (a) The four pulses separated by 7 ns readout by the streak camera and (b) the four pulses each separated by $7+\delta \mathrm{t} n s$.

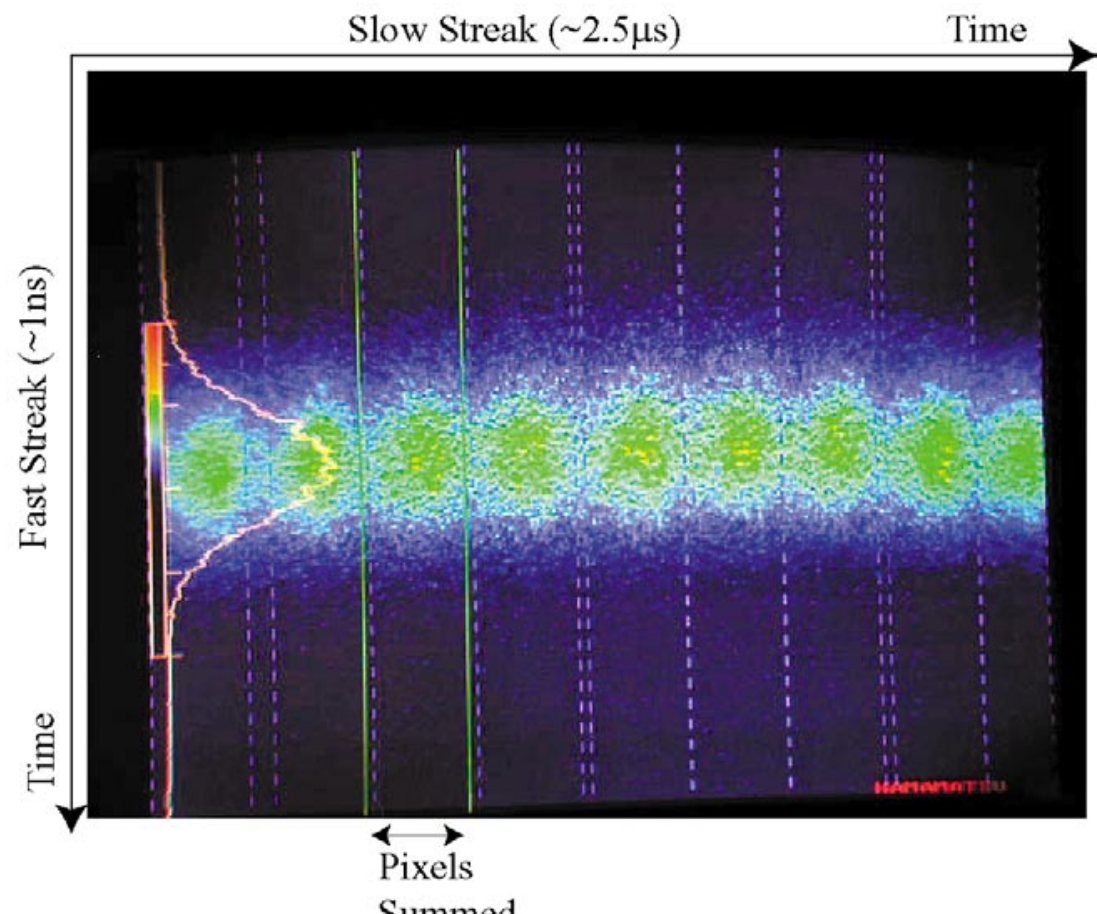

FIG. 7. (Color) This is a sample image of nine positron trains in CESR. Taking a vertical slice and summing the pixels between the slices gives the longitudinal profile shown to the left. The green lines denote the vertical slice that is the summed train. The profile of the distribution is displayed to the left. A profile of the train is displayed in Figs. 13 where the distributions are fit to a Gaussian function. For this image, nine longitudinal profiles were saved. 
With the basic operation of the DSSSC described above, an explanation of the longitudinal dipolecoupled bunch instability as seen by the streak camera is in order. Under normal operation, bunches within a train are separated by $14 \mathrm{~ns}$, which would be two sine waves apart at $143 \mathrm{MHz}$. If they deviate from the $14 \mathrm{~ns}$ spacing, the bunches will have different phases on the sine wave, and this phase difference is detected by the DSSSC. Any instability corresponding to centroid motion in longitudinal phase space can be detected with the DSSSC. An example of a stable and unstable train of bunches is displayed in Fig. 6(a) and (b) when the bunches are separated by $7 \mathrm{~ns}$.

In order to quantify the systematic errors involved with the streak camera, the linearity of the camera was measured during high-energy collisions in CESR. The measurement consists of taking streak camera images of one train and fitting each bunch in the train to an asymmetric Gaussian function to determine the bunch length and asymmetry factor. Images were taken at different trigger settings to determine the linearity of the streak voltage for the synchroscan unit. The bunch length (in terms of pixels) as a function of the mean position on the synchroscan timing without the streak camera linearity is plotted Fig. 8(a). Without the streak voltage calibration, the bunch length is highly correlated with the synchroscan unit timing. The calibration curve for the synchroscan unit is displayed in Fig. 8(b).
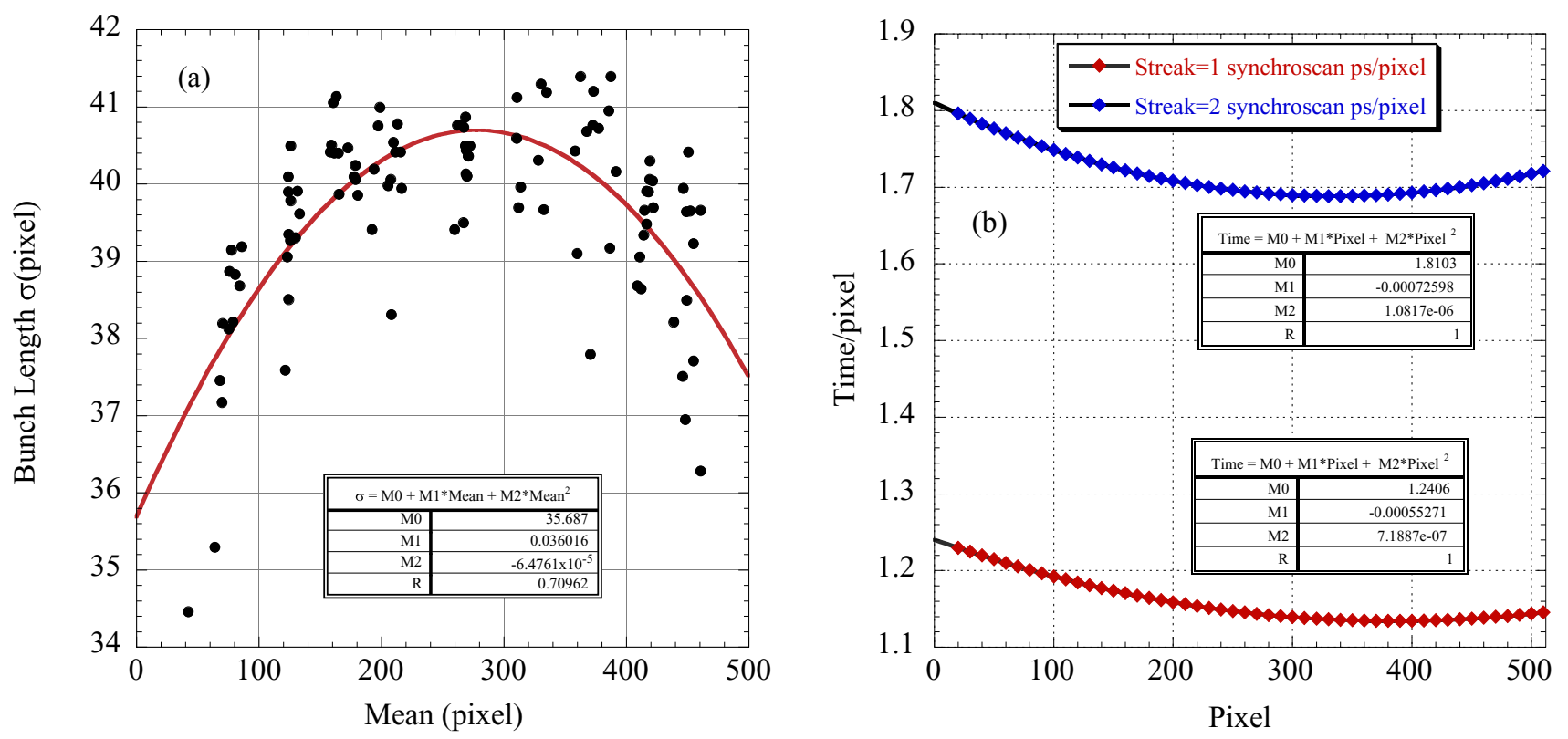

FIG. 8. (Color) (a) The measured bunch length at different trigger settings without the calibration correction and (b) the synchroscan unit calibration curve.

Using the calibration curve from Fig. 8(b), the calibrated bunch length and asymmetry factor are determined. Figure 9(a) is the bunch length and Fig. (b) is the asymmetry factor as a function of synchroscan unit timing. Both the bunch length and asymmetry factors are slightly correlated with the synchroscan unit timing, but the spread in the data is due to statistics and current fluctuations. The bunch length for the different bunches in the train is displayed in Fig. 10. Two comments can be 
made about the data. (i) The bunch length for the last bunch in the train is smaller than the other three. This is due partly to the lifetime in bunch four being lower that results in a lower bunch current for the fourth bunch at the time of the scan. This can explain partly the large differences in the bunch length and asymmetry factor compared to the other bunches. (ii) There are large fluctuations in the bunch length when the timing is at either extreme of the synchroscan timing. Measurements taken in this paper are with the image towards the center of the streak sweep timing.
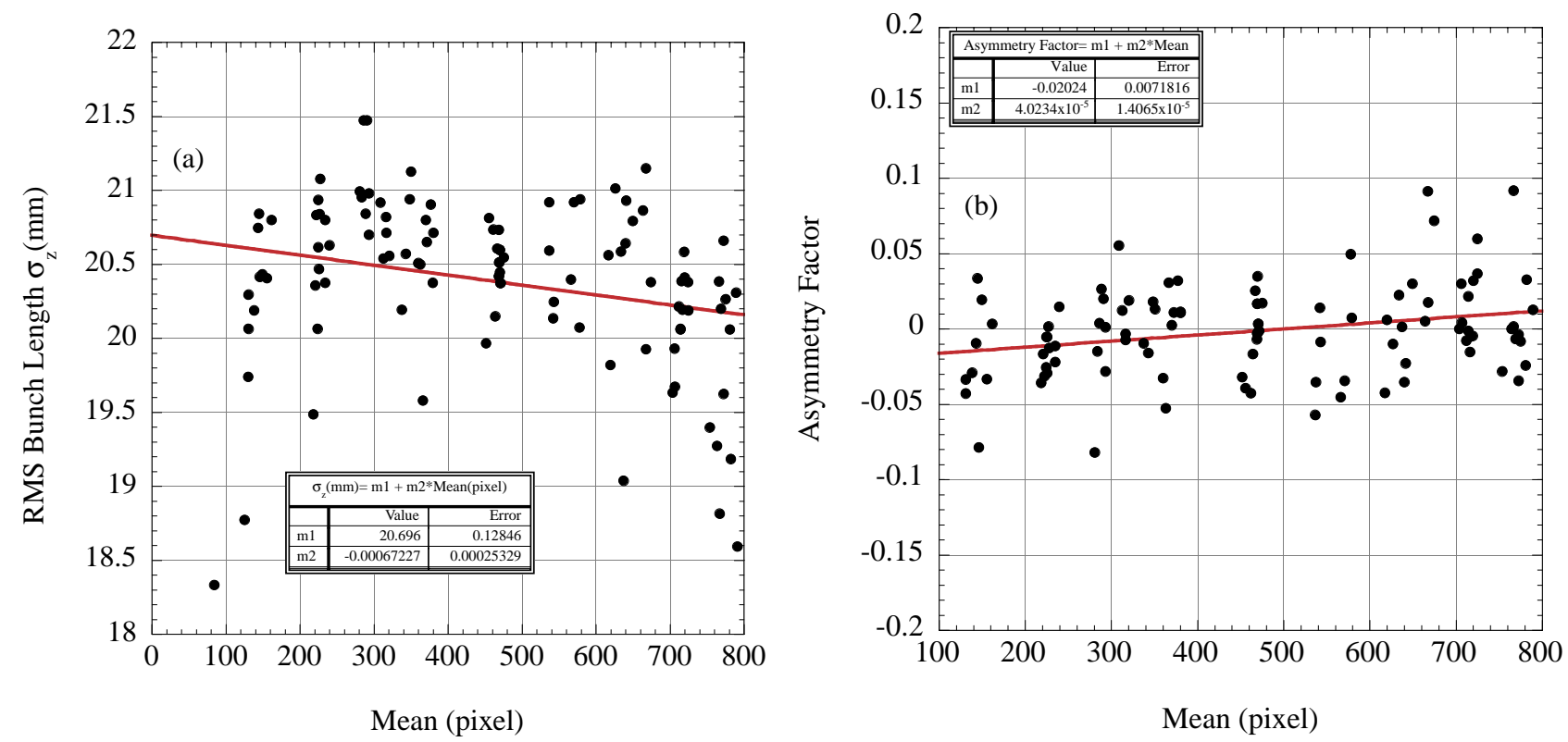

FIG. 9. (Color) (a) The bunch length and (b) asymmetry factor as a function of trigger setting using the synchroscan calibration curve shown in Fig. 8(b).

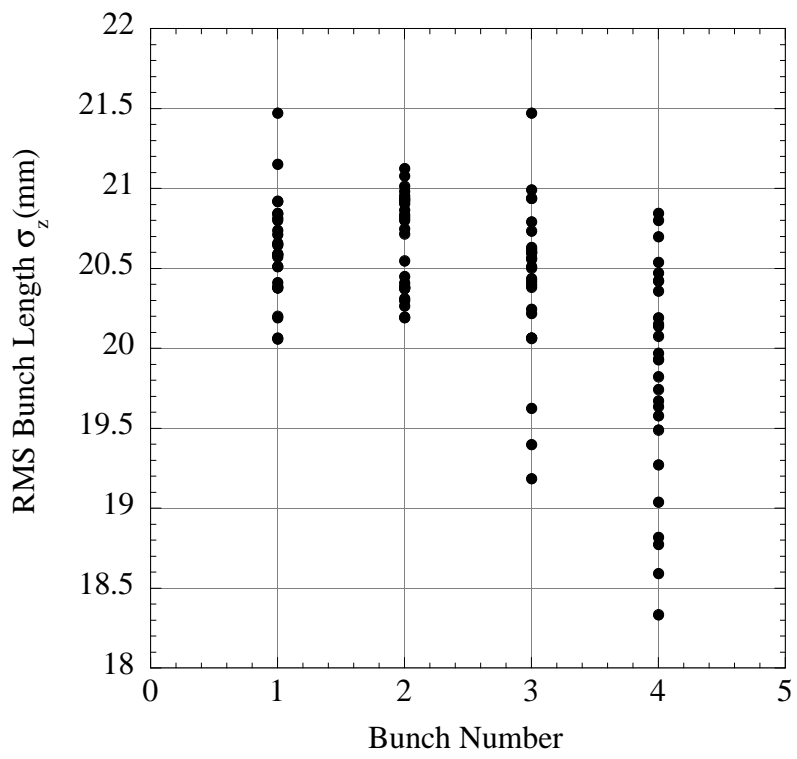

FIG. 10. The bunch length for the four bunches in the train. The mean bunch length for bunch 1 through 4 are $20.6 \pm 0.3 \mathrm{~mm}, 20.7 \pm 0.3 \mathrm{~mm}, 20.4 \pm 0.5 \mathrm{~mm}$, and $19.8 \pm 0.7 \mathrm{~mm}$, respectively. 


\section{Measurement of the Longitudinal Dipole-Coupled Bunch Instability}

The following experiments were performed with the dual sweep streak camera: (i) measurement of a single train consisting of four bunches with the longitudinal dipole-coupled bunch instability present, (ii) measurement of multiple trains with the instability present, and (iii) oscillation of bunches during collisions to quantify the luminosity degradation due to the instability. Above the instability threshold the LDCBI is present, except when the longitudinal feedback system is turned on. During normal operation, the feedback system must remain on. The following experiments were performed during special machine studies times when the feedback system could be turned off to induce the instability.

\section{A. Observations of motion within a single train}

With positrons present in CESR, single train measurements of the longitudinal dipole-coupled bunch instability were performed. Setting the slow streak speed to a full sweep range of $100 \mathrm{~ns}$ allows the single train dynamics to be measured. The measurements were made with nine trains of positrons, with four bunches per train, present in CESR. The bunch spacing in each train was $14 \mathrm{~ns}$. At this bunch spacing, the instability threshold is approximately $180 \mathrm{~mA}$ total or $5 \mathrm{~mA}$ per bunch. Measurements were made at total beam current between $180 \mathrm{~mA}$ and $350 \mathrm{~mA}$.

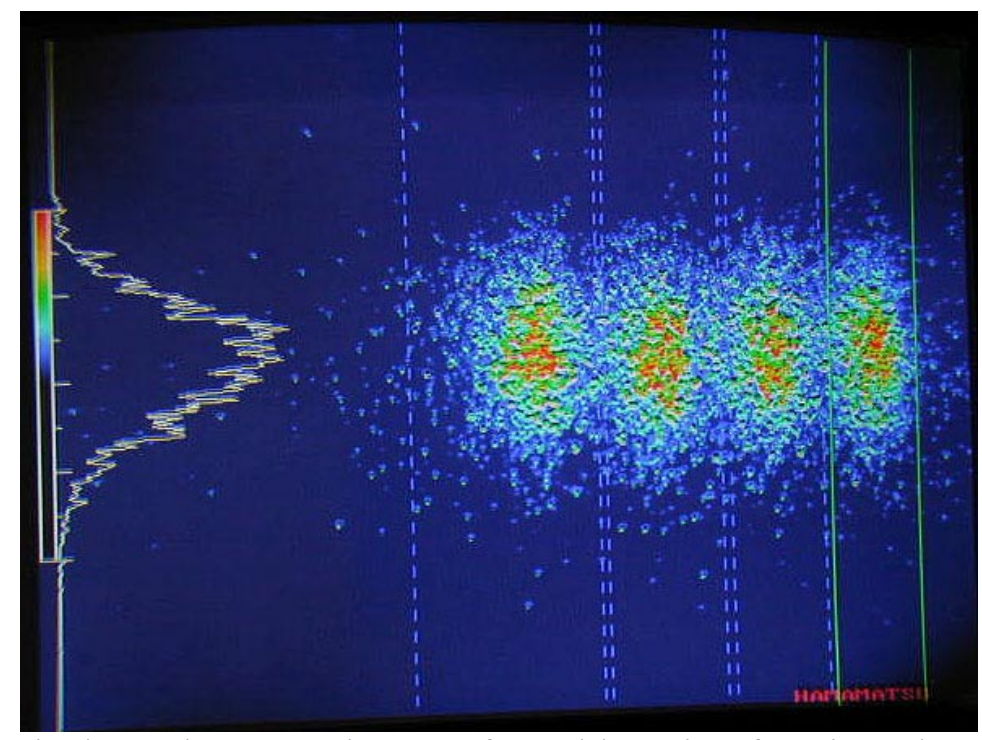

FIG. 11. (Color) A typical streak camera image of a stable train of positron bunches when the longitudinal feedback is turned on.

The presence of the LDCBI was determined by two methods. (i) Above the instability threshold with longitudinal feedback turned off, a signal indicating a dipole instability is observed using a spectrum analyzer. The signature of the instability is synchrotron side bands clearly visible at $\mathrm{f}=\mathrm{nf}_{\mathrm{r}} \pm \mathrm{f}_{\mathrm{s}}$ for some values of $\mathrm{n}$. During this experiment, a spectrum analyzer was used to measure the amplitude and frequency of the beam spectra for $\mathrm{f}=\mathrm{f}_{\mathrm{r}}+\mathrm{f}_{\mathrm{s}}$. The results are listed in Table II. (ii) The 
dual-sweep streak camera can detect the LDCBI. Figures 11 and 12 are images of a single train of bunches without and with the instability present. When the instability is present, the profiles of the four bunches do not align vertically, as shown in Fig. 12. The images with the instability present were chosen due to observed large time oscillations.

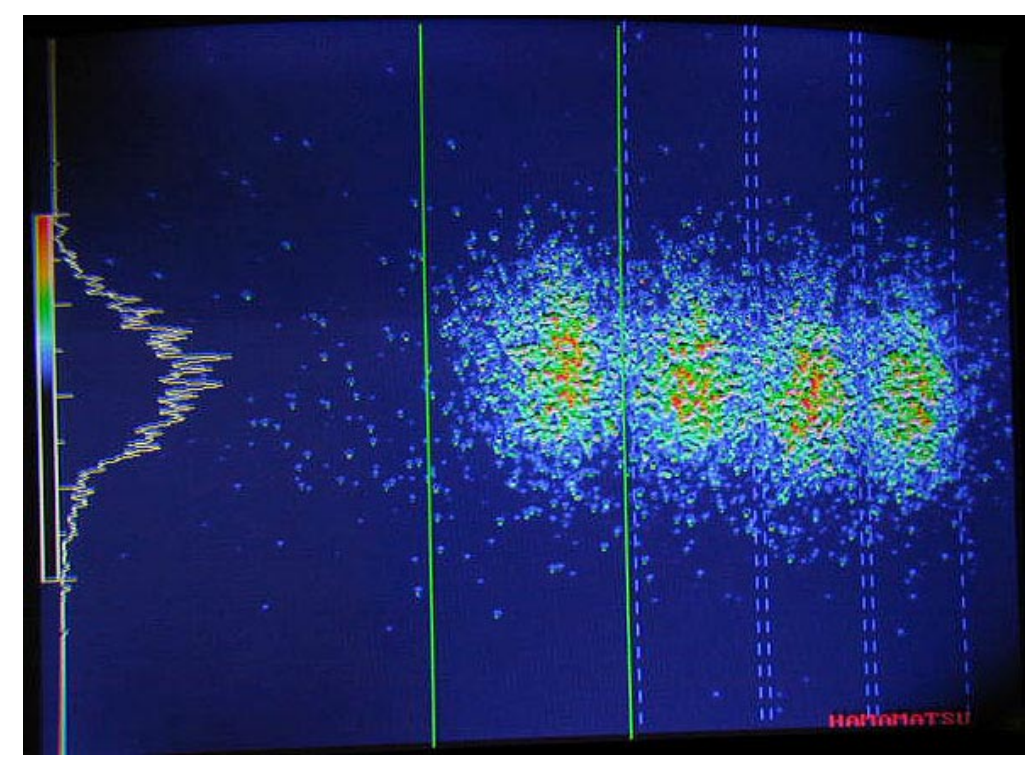

FIG. 12. (Color) A typical streak camera image of a single train of bunches in CESR with the longitudinal feedback turned off and the LDCBI present

\begin{tabular}{|c|c|c|}
\hline \hline Current $(\mathrm{mA})$ & $\mathrm{fr}+\mathrm{fs}(\mathrm{kHz})$ & Amplitude $(\mathrm{dBm})$ \\
\hline \hline $183-181$ & 410.75 & -35.3 \\
\hline $227-224$ & 410.68 & -19.6 \\
\hline $276-272$ & 410.60 & -18 \\
\hline $326-316$ & 410.45 & -31.1 \\
\hline $350-344$ & 410.38 & -28.8 \\
\hline
\end{tabular}

TABLE II. The measured currents, dipole-mode frequency, and intensity for these measurements.

Several single trains images, such as those in Figs. 11 and 12, were made at different beam currents, with and without the instability present. For each image, the longitudinal profile of each bunch in the train was fit to an asymmetric Gaussian function. The amplitude of time oscillation for each bunch is quantified by computing the shift in its centroid (mean) relative to the other bunches in the train. If the bunches are stable, the centers of the four bunch distributions in a train should superimpose in time. If the longitudinal dipole-coupled bunch instability is present, the bunch's centroids oscillate in time, and will not, in general, be aligned in time. In Fig. 13, the longitudinal distributions of bunches in a single train are superimposed for the case when the beam is stable [Fig. 13(a)] and unstable [Fig. 13(b)]. 

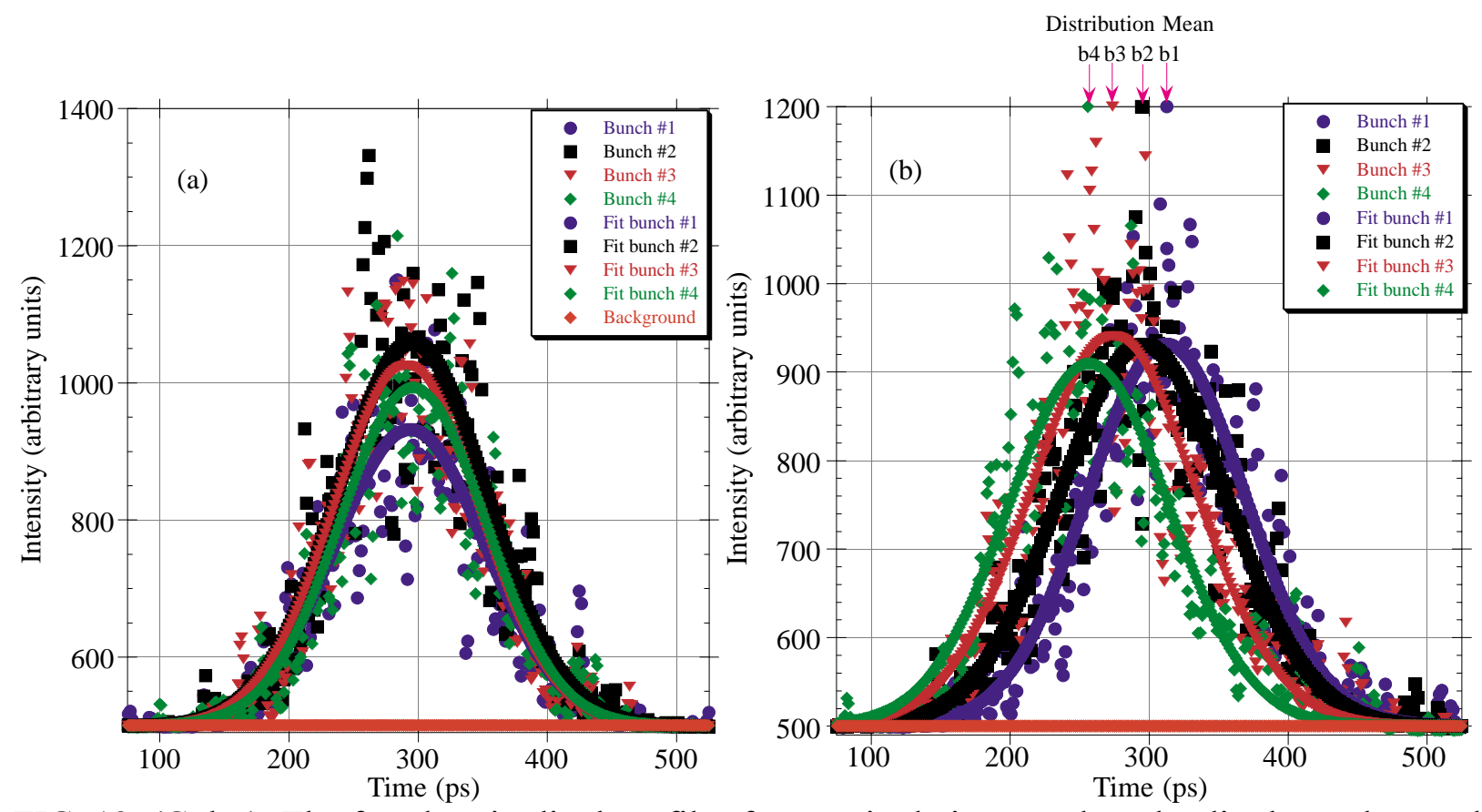

FIG. 13. (Color) The four longitudinal profiles from a single image when the dipole mode coupled bunch instability is (a) not present and (b) present. The mean of the individual distributions when the instability is present is shown above (b). The total positron current when these images were taken was $276 \mathrm{~mA}$.

A spectrum analyzer was used to determine the strength of the LDCBI in conjunction with the streak camera images. As the total beam current increased, the amplitude of the instability increased for the first three measurements $(\mathrm{I}=183-276 \mathrm{~mA})$, and decreased for the last two measurements $(\mathrm{I}=326-350 \mathrm{~mA})$. This drop in amplitude can be attributed to a different mode of oscillation in the bunch train, and will be discussed later.
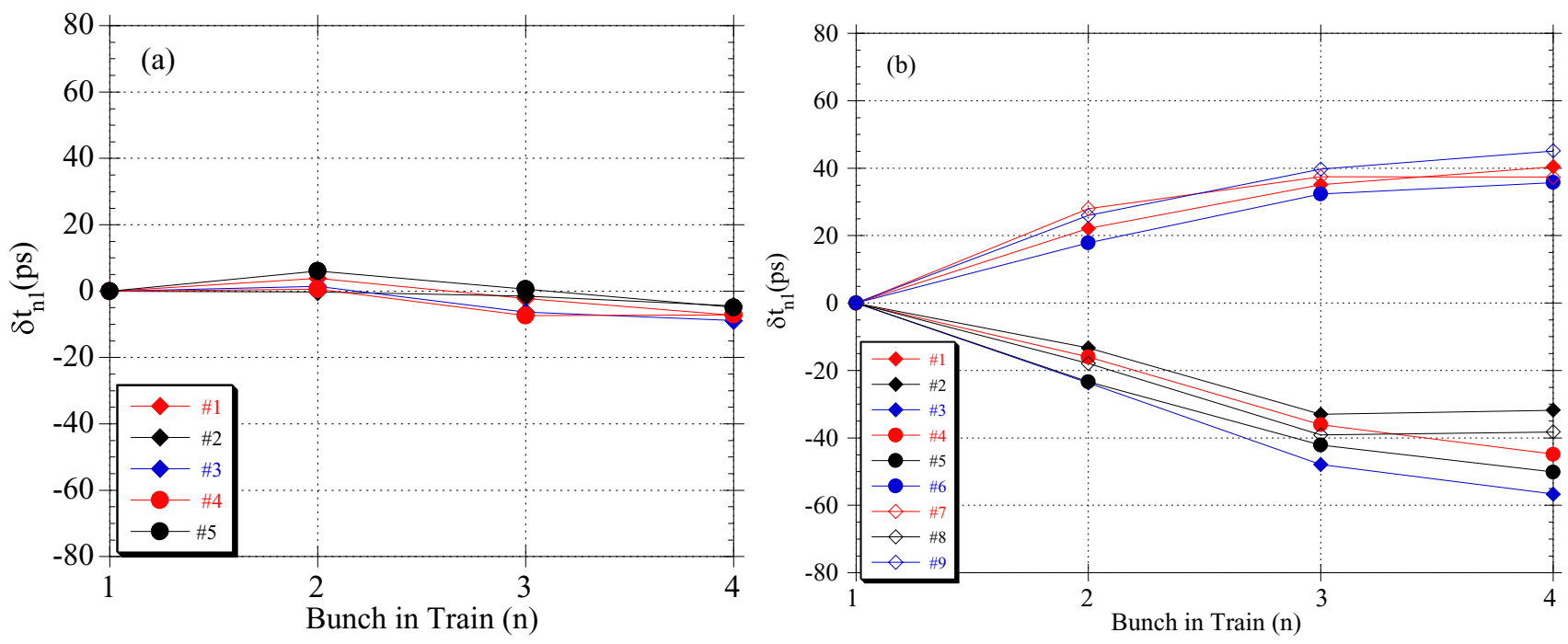


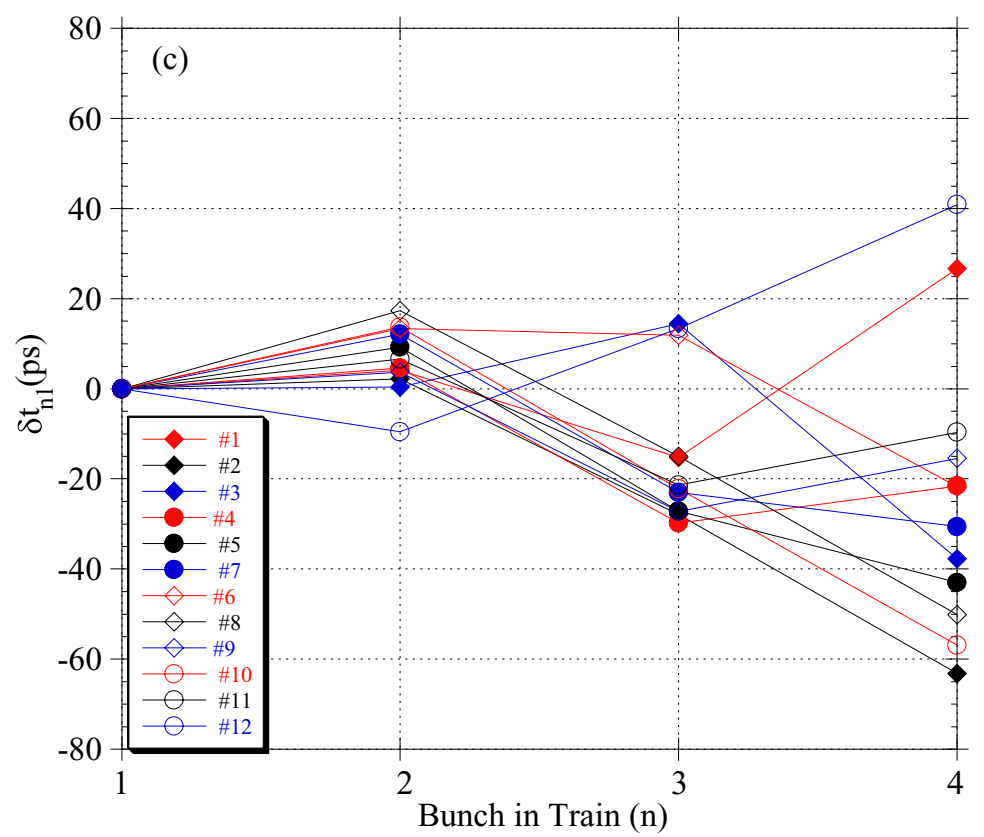

FIG. 14. (Color) The time of arrival oscillation $\left(\delta \mathrm{t}_{\mathrm{n} 1}\right)$ when (a) the train is stable and the total beam current is $276 \mathrm{~mA}$, (b) the train is unstable and the total beam current is $276 \mathrm{~mA}$, and (c) the train is unstable and the total beam current is $326 \mathrm{~mA}$.

Under stable conditions the bunches in a train are separated by $14 \mathrm{~ns}$. When the instability is present, the bunches in a train oscillate about the $14 \mathrm{~ns}$ spacing. The amplitude of the time oscillation is determined from the longitudinal profiles fit to an asymmetric Gaussian function. The centroid position of the bunch is determined from the fit to the data. For our analysis, the amplitude of the time oscillation is referenced to the leading bunch (first bunch in the train). The change in arrival time for the bunches after the leading bunch is given by

$$
\delta \mathrm{t}_{\mathrm{n} 1}=\operatorname{Mean}_{\mathrm{n}}-\operatorname{Mean}_{1} \text { for } \mathrm{n}=2,3,4,
$$

where mean $_{n}$ is the trailing bunch $n$ 's mean centroid position. The sign convention is the following: A positive $\delta t_{n 1}$ means the trailing bunch arrives $(n-1) 14 n s+\delta t_{n 1}$ after the leading bunch in a train. The time deviation from the lead bunch is displayed for a number of sample triggers in Fig. 14 for each of three different cases: (a) when the train was stable at a current of $276 \mathrm{~mA}$, (b) when the train was unstable at a current of $276 \mathrm{~mA}$, and (c) when the train was unstable at a current of $326 \mathrm{~mA}$.

Several comments can be made about the results. (i) Using this analysis technique it is easy to see that the bunches in a train oscillate in time. The longitudinal feedback system stabilizes the bunch time oscillations to a few picoseconds. When turned off, the time oscillation has been measured to approximately $100 \mathrm{ps}$. The unstable time oscillations substantially degrade the high-energy physics luminosity. (ii) The data suggest that a change in bunch oscillation mode occurs between $276 \mathrm{~mA}$ and $326 \mathrm{~mA}$. At $276 \mathrm{~mA}$ and below, all the bunches move closer, or further away in unison, according to Fig. 14(b). This motion, from now on referred to as "accordion" oscillation, is displayed in Fig. 4(b) as unstable mode 1, and is detected in the Fig. 12 streak camera image. At $326 \mathrm{~mA}$ and above, the accordion oscillation changes according to Fig. 14(c). The second bunch in the train 
oscillates in the opposite direction as the third and fourth bunch. This mode of oscillation is depicted in Fig. 4(c) as unstable mode 2, and is evident in the streak camera image shown in Fig. 15. These measurements, in conjunction with the spectrum analyzer results, reveal that the change in oscillation mode occurs between 276 and $326 \mathrm{~mA}$.

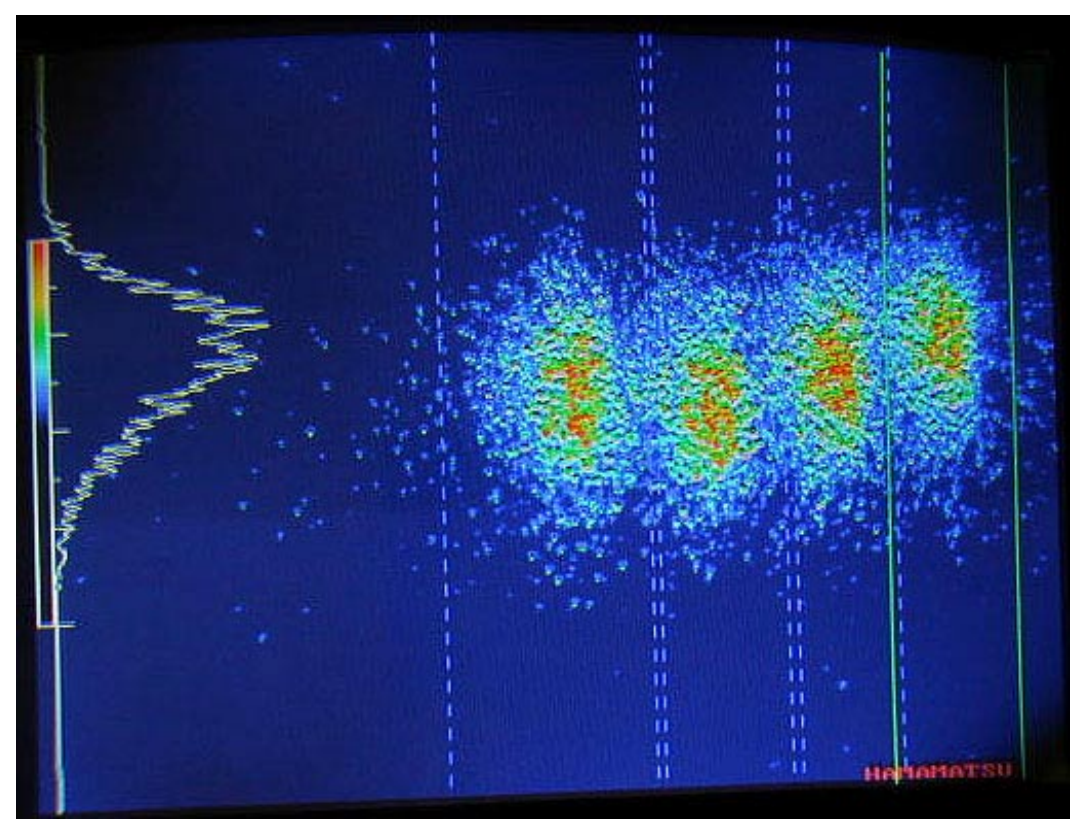

FIG. 15. (Color) A typical streak camera image taken with $326 \mathrm{~mA}$ of positrons present in CESR. The second bunch in the train arrives later than the first bunch. This signifies a change in the instability's oscillation mode.

(iii) The oscillation amplitude limits in the presence of the instability. Although the mechanism for this limiting has not been proven, the nonlinearity of the longitudinal restoring force is the likely candidate. Notice in Table II that above the instability threshold the detected spectral amplitude grows slightly with current and is consistent with the oscillation amplitude remaining constant and the signal amplitude increasing proportional to the beam current. This continues until the mode of oscillation changes. (iv) The dipole mode signal was the only signal observed by the spectrum analyzer during these measurements, and, in particular, there were no signals indicating high modes of oscillation.

The time deviation of adjacent bunches due to the LDCBI can be defined as

$$
\mathrm{T}_{\mathrm{mn}}=\mid \text { mean }_{\mathrm{m}}-\text { mean }_{\mathrm{n}} \mid \text {, }
$$

where mean $_{m}$ and mean $_{n}$ are the mean centroid positions of adjacent bunches in the train. The results of this calculation are displayed in Fig. 16.

The results suggest that: (i) When the bunches are stable, the average time deviation between bunches averages $3.4 \mathrm{ps}$ for $\mathrm{T}_{12}, 4.5 \mathrm{ps}$ for $\mathrm{T}_{23}$, and $4.0 \mathrm{ps}$ for $\mathrm{T}_{34}$. When unstable, the average time deviation is 11.6 ps for $T_{12}, 19.6$ ps for $T_{23}$, and 15.4 ps for $T_{34}$ suggesting that the bunches move in synchronous phase relative to one another on the average of 10 to 15 ps over the current range of 180 
to $330 \mathrm{~mA}$. This might suggest a systematic change in bunch length through each train or some large change in the beam's wakefield. (ii) At the two highest currents, the average time deviations, $\mathrm{T}_{23}$ and $\mathrm{T}_{34}$, increase while $\mathrm{T}_{12}$ decreases. This jump is another signature of a change in the character of the LDCBI oscillation mode.

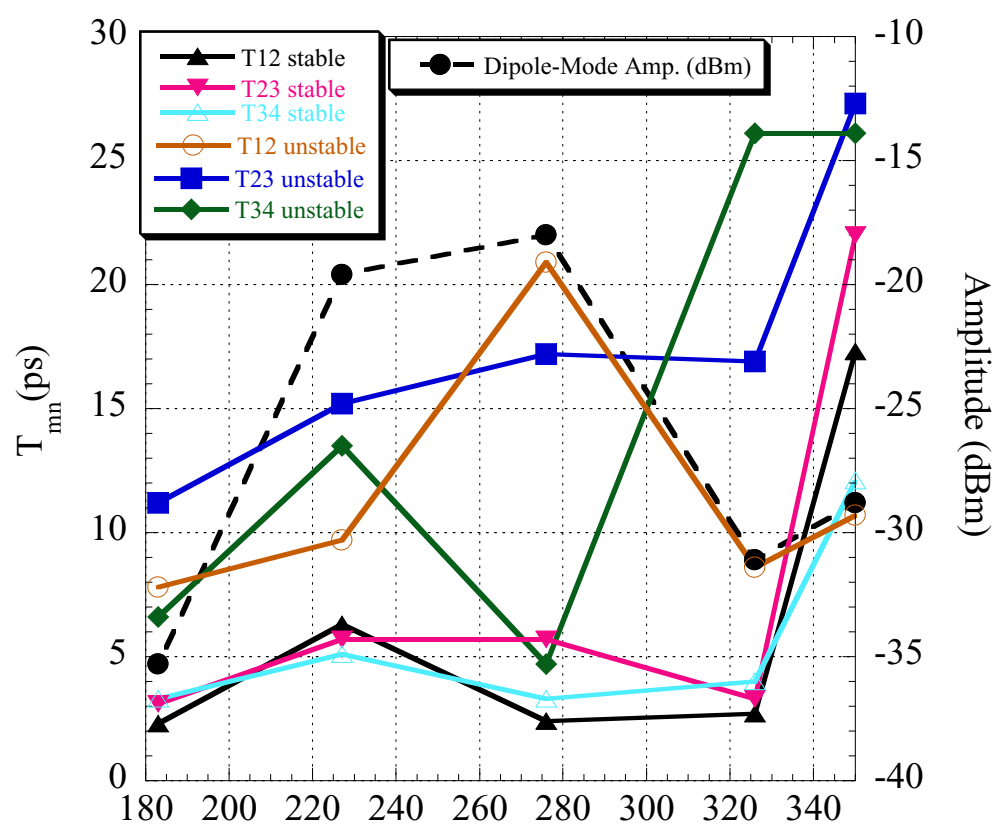

Total Current (mA)

FIG. 16. (Color) The time deviation between adjacent bunches in a train as a function of current, with and without the instability present.

In studies that were made when only normal conducting copper cavities were present in CESR, oscillations having the same spectral characteristics as longitudinal quadrupole-coupled bunch oscillations were detected by exciting the dipole-mode to large amplitude [3]. The presence of these quadrupole-mode-like oscillations implies a change in the bunch distribution and has been observed to lead to beam loss. As explained earlier, the shape in the bunch distribution does change for large amplitude oscillations. Since it was shown that the asymmetry factor of a bunch is expected to be an odd function of the temporal displacement of the bunch due to the rf system's longitudinal restoring force, a linear dependence of the asymmetry factor on the temporal displacement of the bunch will be initially assumed. The larger the time deviation behind the first bunch in the train, the larger the asymmetry factor deviates from the head bunch's asymmetry factor. This assumption may be tested by correlating the time deviation with the asymmetry deviation. The difference in asymmetry factor between the first bunch in the train and subsequent bunches will be defined as

$$
\delta \mathrm{A}_{\mathrm{n} 1}=\mathrm{A}_{\mathrm{n}}-\mathrm{A}_{1} \text { for } \mathrm{n}=2,3,4,
$$

where $A_{n}$ is the asymmetry factor for the trailing bunch $n$. By taking the difference in asymmetry factor between bunches any constant change in the symmetry of the bunch's distribution due to potential well distortion can be ignored. Figures 17(a) and 17(b) are typical, measured bunch 
distributions that have large time deviations. Notice also that there are large asymmetry factors present for the fits to the distribution.
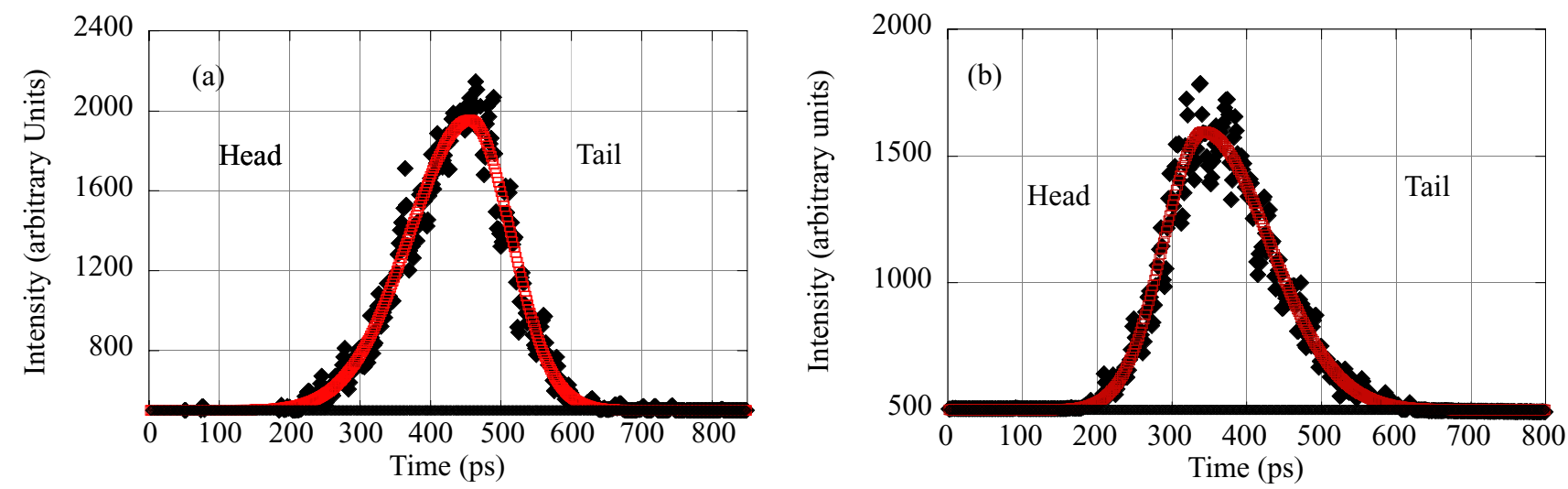

FIG. 17. (Color) Two single streak camera distributions fit to an asymmetric Gaussian function where the asymmetry factor and time deviation between bunches is (a) $\delta \mathrm{As}_{31}=0.2$ and $\delta \mathrm{t}_{31}=79 \mathrm{ps}$, (b) $\delta \mathrm{As}_{41}=-0.23$ and $\delta \mathrm{t}_{41}=-102 \mathrm{ps}$.

The correlation between the time deviation, $\delta \mathrm{t}_{\mathrm{n} 1}$, and the difference in asymmetry factor is seen in Fig. 18 with these measurements taken in the presence of the dipole instability.

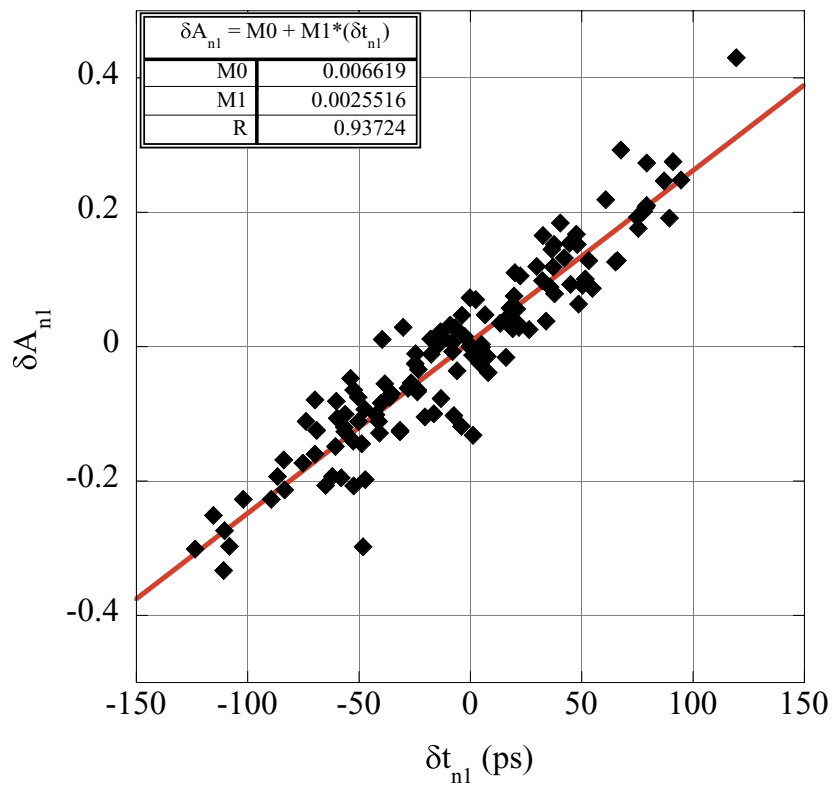

FIG. 18. (Color) A plot of the change in the asymmetry factor from bunch one to bunch $\mathrm{n}$ as a function of time deviation from bunches one to bunch $\mathrm{n}$ in the presence of the longitudinal instability.

The fact that the time deviation and the difference in the asymmetry factor are highly correlated permits drawing some conclusions: If the bunches are spaced by $7 \mathrm{rf}$ wavelengths (14 ns), they are called synchronous and they line up on the vertical axis of the streak camera images. When bunches are unstable, the bunches, trailing the lead bunch, oscillate about this synchronous location [Fig. 6(b)]. In the presence of this oscillation the bunch asymmetry factor changes linearly as a function of the 
temporal displacement, so that if the oscillating bunch arrives early, the length of the tail of the bunch grows; if it arrives late, the length of the head of the bunch grows.

The wakefield of a bunch produces a voltage which is felt by subsequent bunches. This voltage is typically modeled as being largest within the bunch (the self-wake), and after the bunch it will vary in magnitude and sign as the sum of many damped oscillators which have frequencies below the beam pipe's cutoff frequency (approximately $3 \mathrm{GHz}$ in CESR). Since it is the largest of the wakefield voltages, and since it remains locked to the temporal position of the bunch, the self-wake has the largest effect, that of distorting the bunch's potential well and, hence, the shape of the distribution. (This effect was already presented in the bunch lengthening seen in Fig. 10, and the associated remarks that the last bunch had such a large variation in bunch length is likely due to the large variation in current of the last bunch in the trains [8].) A simple way to view the effect of the wakefield on subsequent bunches is to perform a Taylor series expansion of the wakefield about the equilibrium position of the bunch. The constant term will be the average voltage seen by the trailing bunch due to the preceding bunch. The linear term will give the voltage gradient. When the preceding bunch is undergoing small dipole oscillations, the succeeding bunches will feel a time varying dipole kick proportional to the slope of the wakefield voltage, a time varying change in slope (which would drive quadrupole oscillations) proportional to the second derivative of the wakefield voltage, and so on. When a dipole instability is present, the oscillation of the bunches will drive subsequent bunches by their wakefield coupling. Typically, the effect of these wakefield kicks is small on a turn-by-turn basis, but, because the bunches are being excited near their resonant frequency for synchrotron motion, the oscillation amplitude builds up over many turns. In CESR the instability's growth time is usually many thousands of turns. When a dipole instability is present, the wakefield seen by later bunches is oscillating near the dipole frequency and will not resonantly drive any quadrupole (bunch shape oscillations), so the change in symmetry of the shape of the bunch as a function of temporal displacement must be due to the large dipole amplitude of oscillation interacting with the nonlinearities of the rf system's restoring force.

Last, the correlation between the change in asymmetry factor and time deviation between the lead and trailing bunch, shown in Fig. 18, is evidence of charge flow within the bunch distribution. This flow of charge within the bunch will henceforth be referred to as the distribution is undergoing some form of "breathing". To calculate the degree of breathing in a distribution of particles, consider a pure Gaussian distribution (asymmetry factor $=0$ ), which has an equal amount of charge in the head and tail of the distribution. Consider a distribution that is changed from a pure Gaussian to an 
asymmetric Gaussian distribution that has sustained longitudinal dipole oscillations at large amplitudes. The fact that the distribution is asymmetric together with the fact that the particles in the bunch are rotating in phase space about the equilibrium point causes the distribution to appear to breathe. By superimposing two normalized asymmetric Gaussian distributions with different asymmetry factors, the residual between distributions is determined. Figures 19(a) and 19(b) are examples of two superimposed distributions. The breathing, between the head and tail, with different asymmetry factors is determined by summing the residual in the head and tail of the distribution. A summary of the calculation is shown in Fig. 20(a). To quantify the degree of breathing, as a function of time deviation, Figs. 18 and 20(a) are used and the result is plotted in Fig. 20(b).
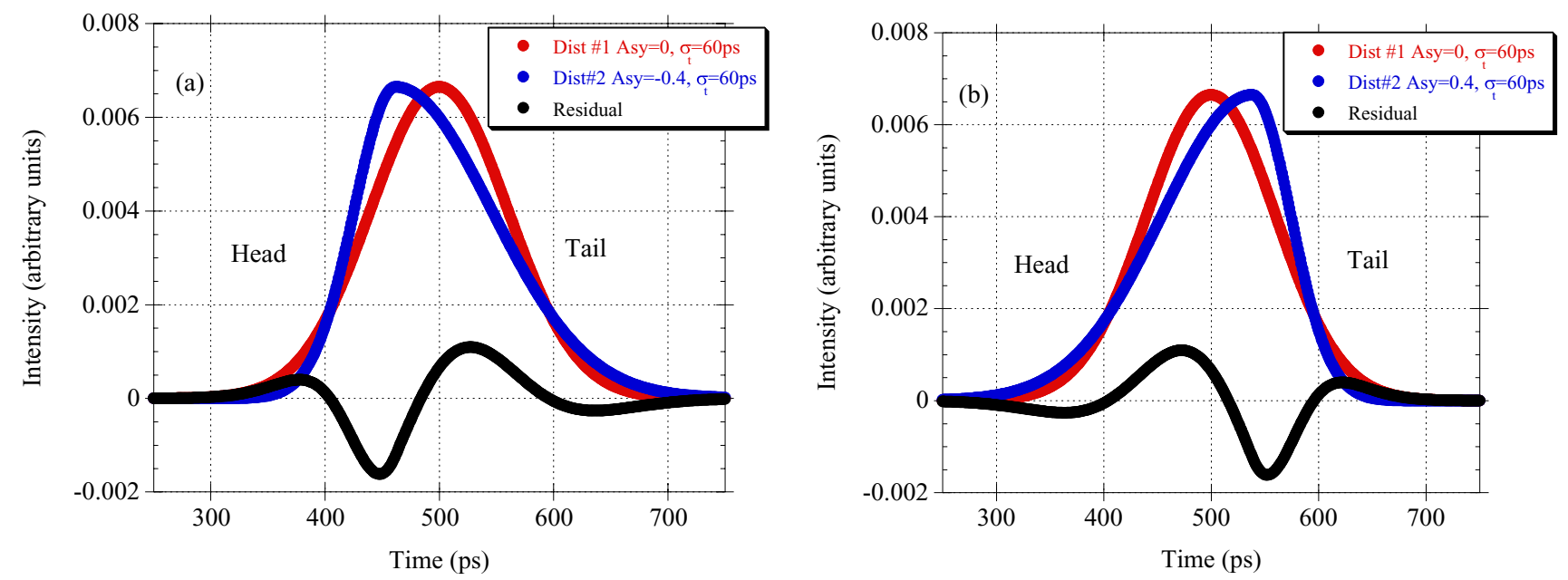

FIG. 19. (Color) Two asymmetric Gaussian distributions with the same bunch length and asymmetry factors of (a) $A_{1}=0$ and $A_{2}=-0.4$, and (b) $A_{1}=0$ and $A_{2}=0.4$. The residual between the two distributions is also displayed.

As an example, if a bunch in a train has a $\tau= \pm 100 \mathrm{ps}$ time deviation from the lead bunch, which corresponds to a change in the asymmetry factor of $+0.26(100 \mathrm{ps})$ and $-0.25(-100 \mathrm{ps})$, the fractional change in charge between the head and tail is approximately 3\%. Figures 21(a) and 21(b) show the change in distribution (assuming the lead bunch is Gaussian) for a $+/-100$ ps oscillation.

The following conclusions can be made from the above analysis: (i) Trailing bunches in a train oscillate in time behind the lead bunch due to the dipole instability. This time oscillation leads to breathing between the head and tail of the trailing bunch distribution. This breathing occurs at the synchrotron frequency. (ii) The degree of breathing depends upon the amplitude of time deviation from the lead bunch. The bunch distribution changes shape as a result of this distribution breathing. This change in the distribution's shape at large dipole oscillation amplitudes may contribute to the fact that the oscillation amplitude limits, since this affects the way that the self-fields react back on the bunch. 

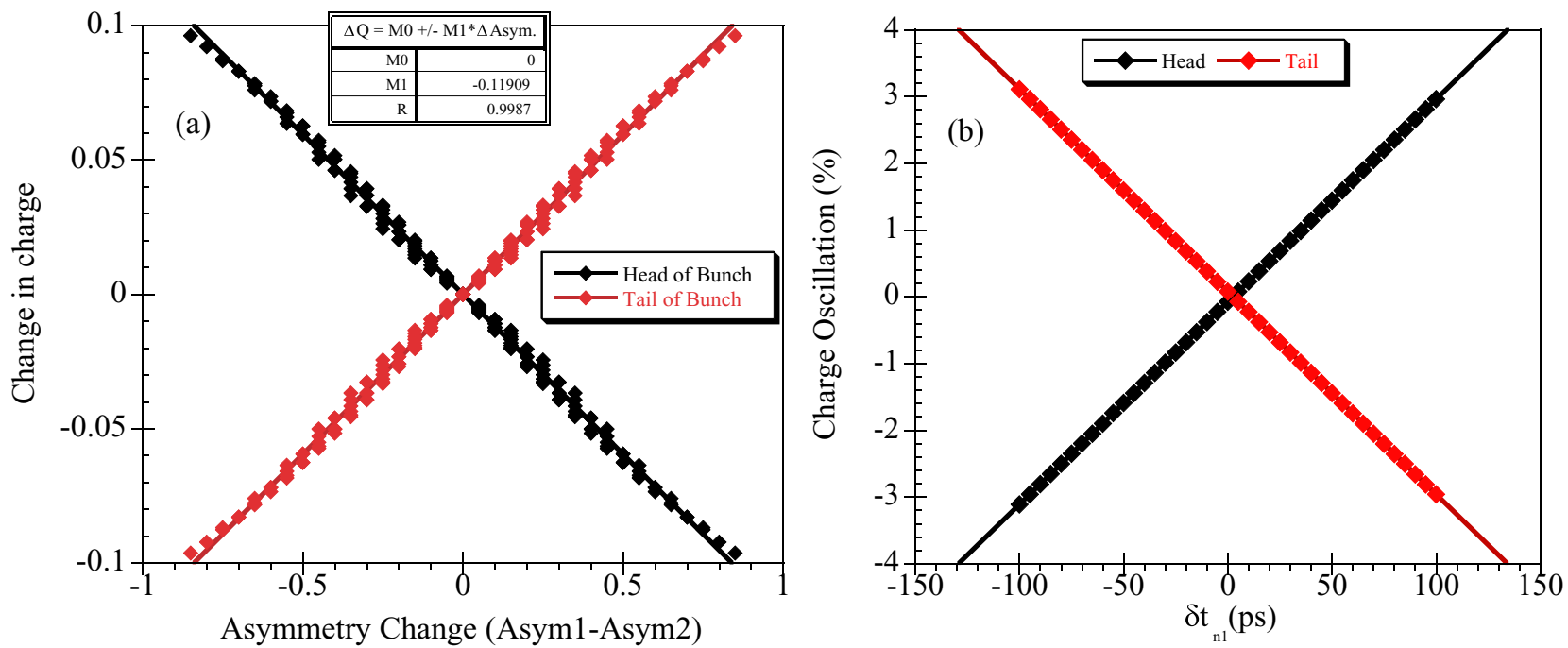

FIG. 20. (Color) (a) The degree of breathing due to an asymmetry factor deviation between two distributions. (b) The degree of breathing in a distribution due to a time oscillation from the lead bunch in a train.
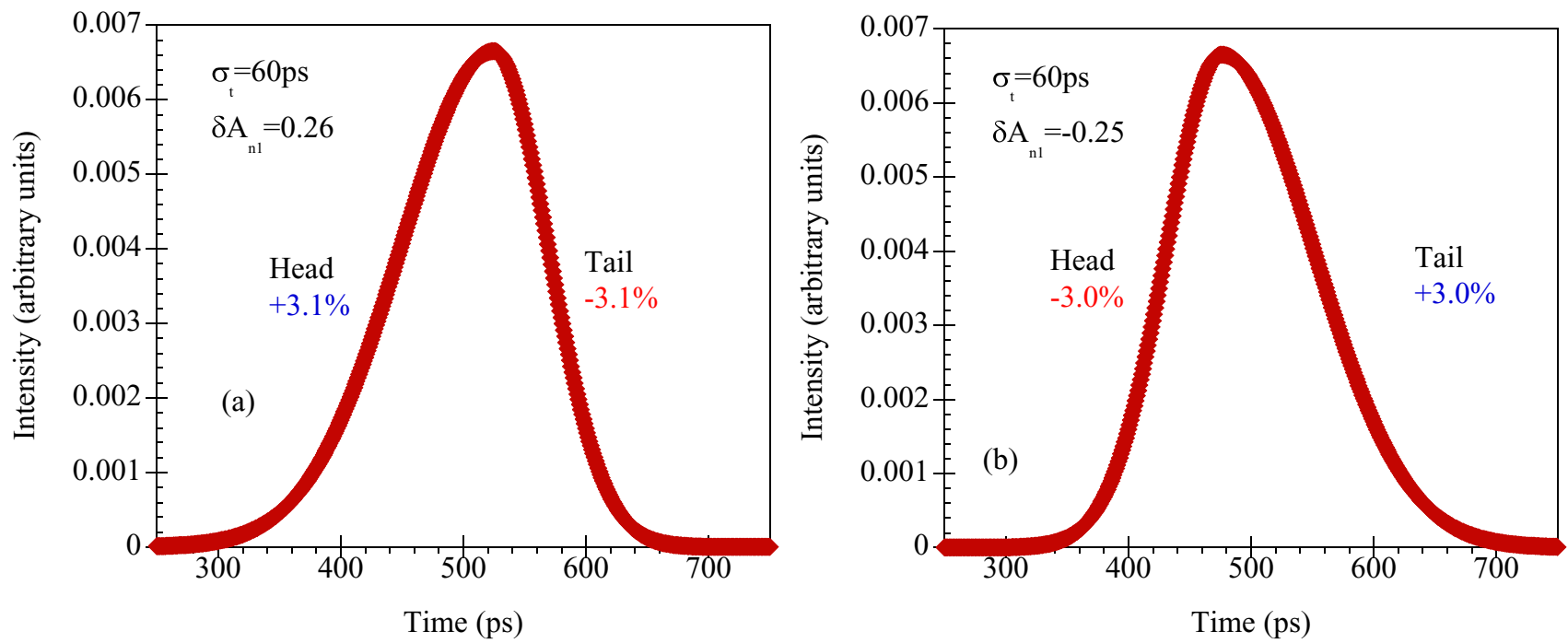

FIG. 21. (Color) The change in the trailing bunches in a train (from a Gaussian distribution) due to a (a) $100 \mathrm{ps}$ deviation and (b) $-100 \mathrm{ps}$ deviation from the lead bunch in the train.

The effect of the longitudinal temporal displacements between colliding electron and positron bunches on luminosity may be estimated for head-on collisions (rather than angle collisions) with the geometric luminosity suppression factor [Eq. (3)], $\mathrm{S}_{\mathrm{f}}$, using the present values of $\beta_{\mathrm{v}}^{*}$ and bunch length. Extrapolating (slightly) a fit of Milton's calculation to include the value of $\mathrm{S}_{\mathrm{f}}$ when the bunch missed colliding in time by $1 \sigma(18 \mathrm{~mm}$ or $60 \mathrm{ps})$ gives a value of 0.61 for $\mathrm{S}_{\mathrm{f}}$ [7]. By comparison, bunches colliding in time have a value of $S_{\mathrm{f}}$ of 0.81 . Therefore, a $1 \sigma$ oscillation of the collision time of bunches yields a geometric reduction factor for the luminosity of 0.75 . In addition to the geometric effects, one would expect the appearance of additional beam-beam dynamical effects that can further 
reduce the luminosity and the lifetime. The presence of large longitudinal oscillation amplitudes in Fig. 18 is suggestive of a very significant reduction in luminosity.

The longitudinal profiles for the four bunches in a train are summed to provide a profile of the train. Each train profile is fit to the asymmetric Gaussian function. The average time deviation, which is the average time the three trailing bunches deviate from the leading bunch, is computed. An interesting correlation can be made between the average train deviation and the train asymmetry factor (Fig. 22). If all the bunches arrive early, the train has a long tail, and a long head for late arriving trains. Figure 23 displays a schematic description of the longitudinal dynamics. A negative time deviation represents an early arrival. A train with a negative asymmetry (long tail) and positive asymmetry is shown in Fig. 23. A more detailed study of the dynamics in a train will follow in the next section.

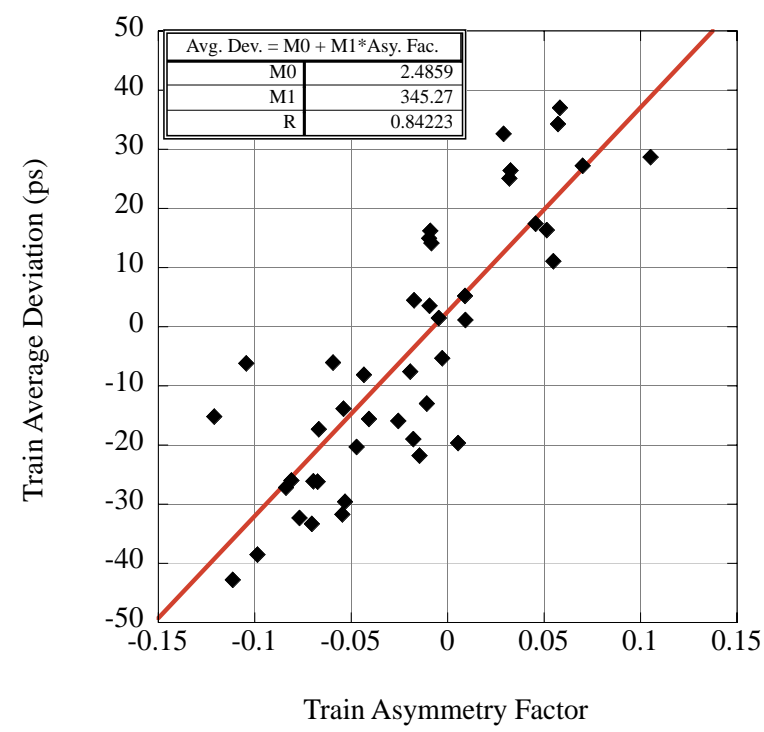

FIG. 22. (Color) A correlation between the train asymmetry factor and the average time deviation.

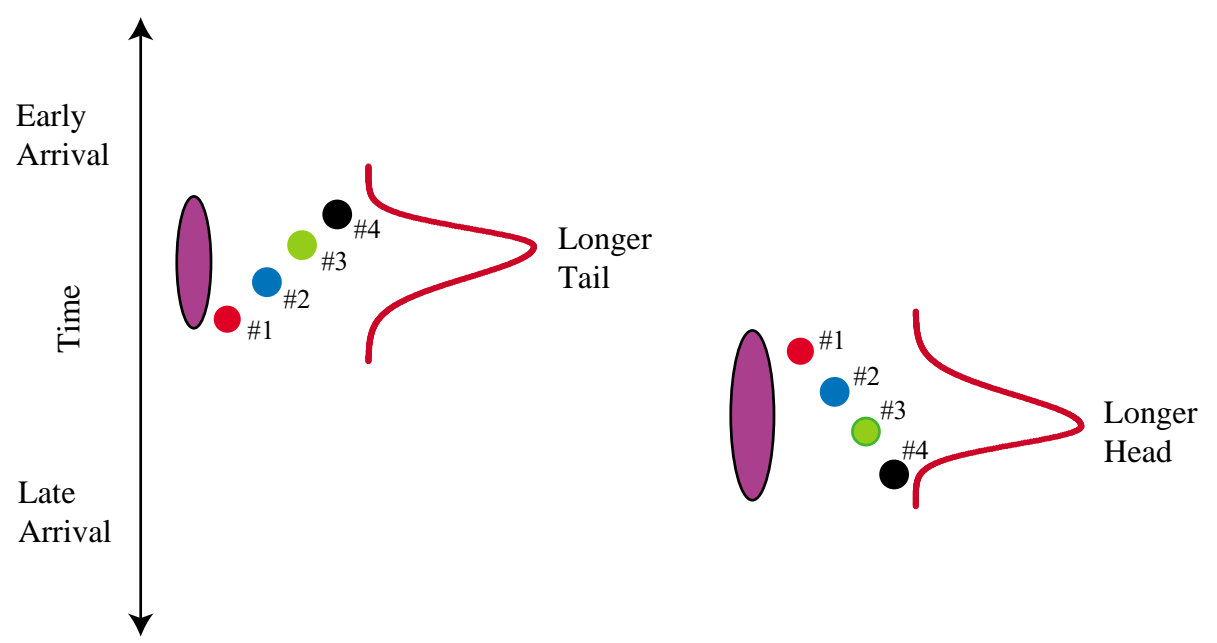

FIG. 23. (Color) This figure displays the dynamics of the bunches in a train. A positive time deviation means the bunches in a train arrive late and the train bunch distribution has a longer head. The mode of oscillation is described for current between 183-276 mA when the oscillation mode is the accordion oscillation. 


\section{B. Observation of multiple trains}

Up to this point, we have concentrated on bunch motion within a single train and have ignored train-to-train dynamics. By reducing the speed of the slow streak plane, multiple trains can be observed, with the limitation that bunches within the train cannot be resolved. The measurements presented in this section were made with positrons present in CESR. Figures 24 and 25 show typical images taken with the dual-sweep streak camera when nine trains of four bunches per train were present in CESR under both stable and unstable conditions. Each train pulse shown in these figures consist of four bunches, and the mean of the train will be referred to as the centroid of the train of four bunches.

As was shown in the previous section, bunches within a train oscillate in time when the longitudinal dipole-coupled bunch instability is present, and it is evident from these images (Figs. 24 and 25) the train centroids also oscillate with the instability present. These two different modes of oscillation may be classified as bunch modes and train modes [9]. From these images the amplitude of time oscillation for each train was determined by fitting each train profile to an asymmetric Gaussian function. The longitudinal centroid of each train is determined from the fit to the data. For each image, which consists of nine trains, the image centroid and standard deviation are calculated. If an oscillation is present, the standard deviation will have a component due to bunch length and another component proportional to the dipole oscillation amplitude. For sinusoidal oscillation of the bunch's arrival time, the total standard deviation $\sigma_{\mathrm{t}}$ over many observations will be given by

$$
\sigma_{\mathrm{t}}^{2}=\sigma_{\mathrm{b}}^{2}+\frac{1}{2} \hat{\mathrm{z}}^{2}
$$

where $\sigma_{\mathrm{b}}$ is the bunch length and $\hat{z}$ is the longitudinal spatial oscillation amplitude.

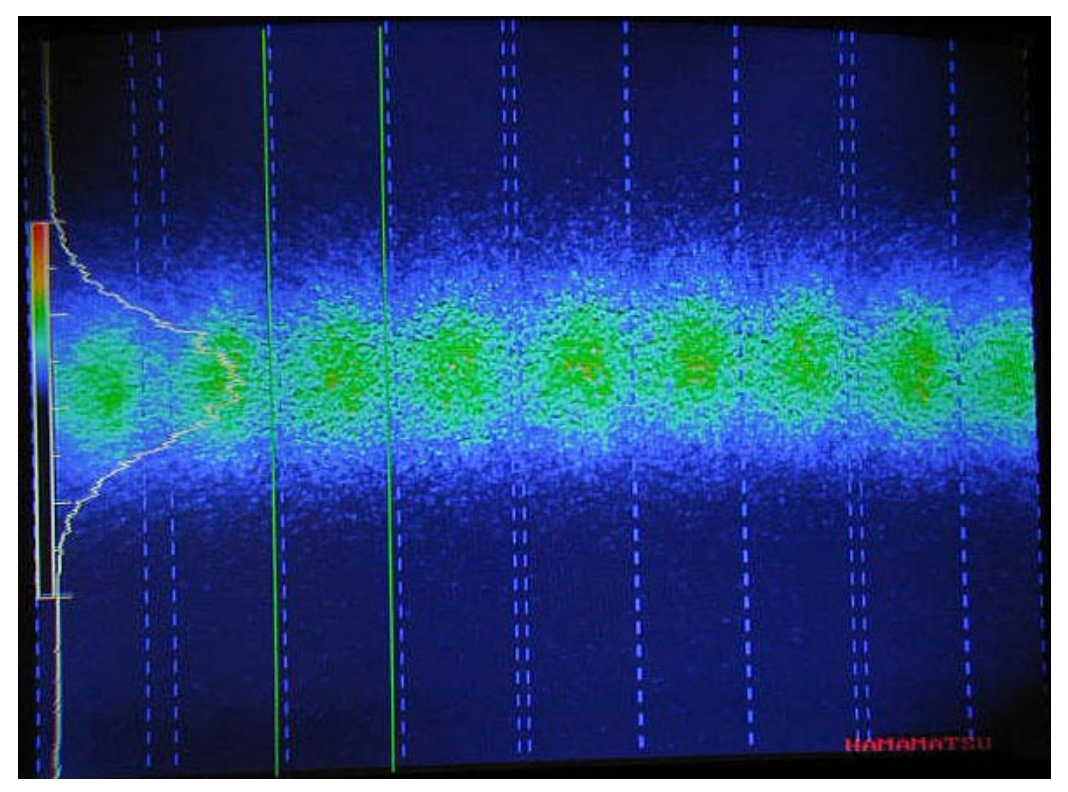

FIG. 24. (Color) A typical image of nine stable trains of bunches of positrons in CESR. The total beam current for this measurement was $184 \mathrm{~mA}$. 


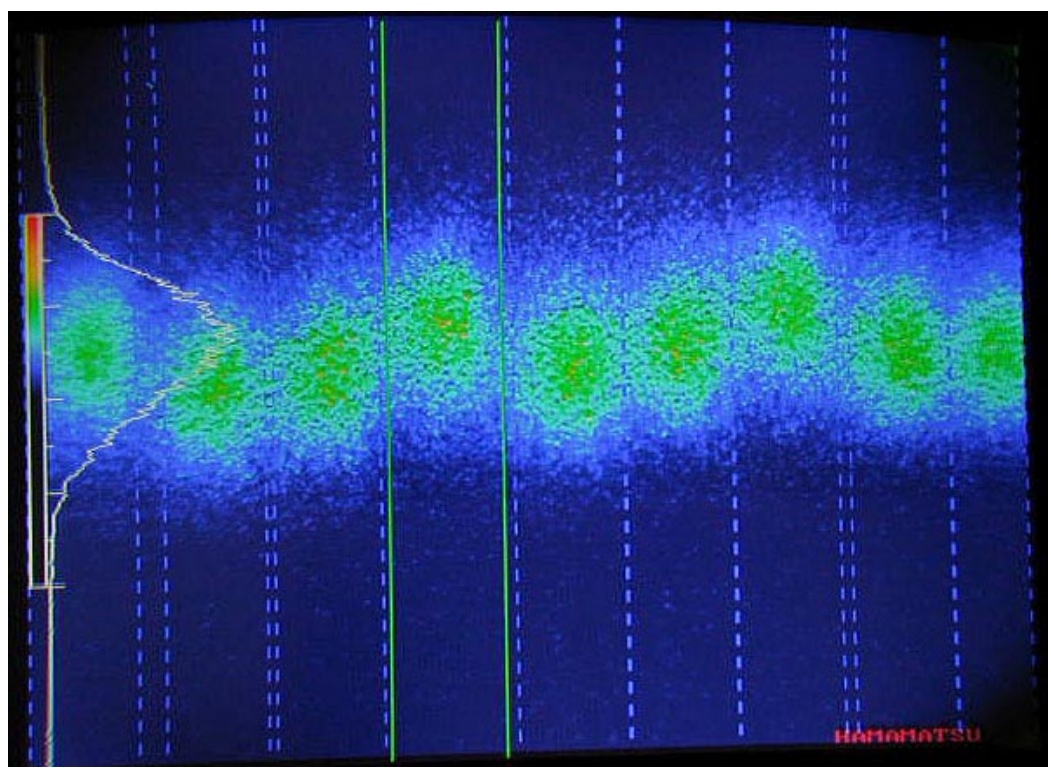

FIG. 25 (Color) A typical image of nine unstable bunches of positrons in CESR. The total beam current for this measurement was $184 \mathrm{~mA}$.

Figure 26(a) is a typical train profile fit to an asymmetric Gaussian function. Figure 26(b) is the mean of the standard deviation of train's distributions at each current with and without the instability present.
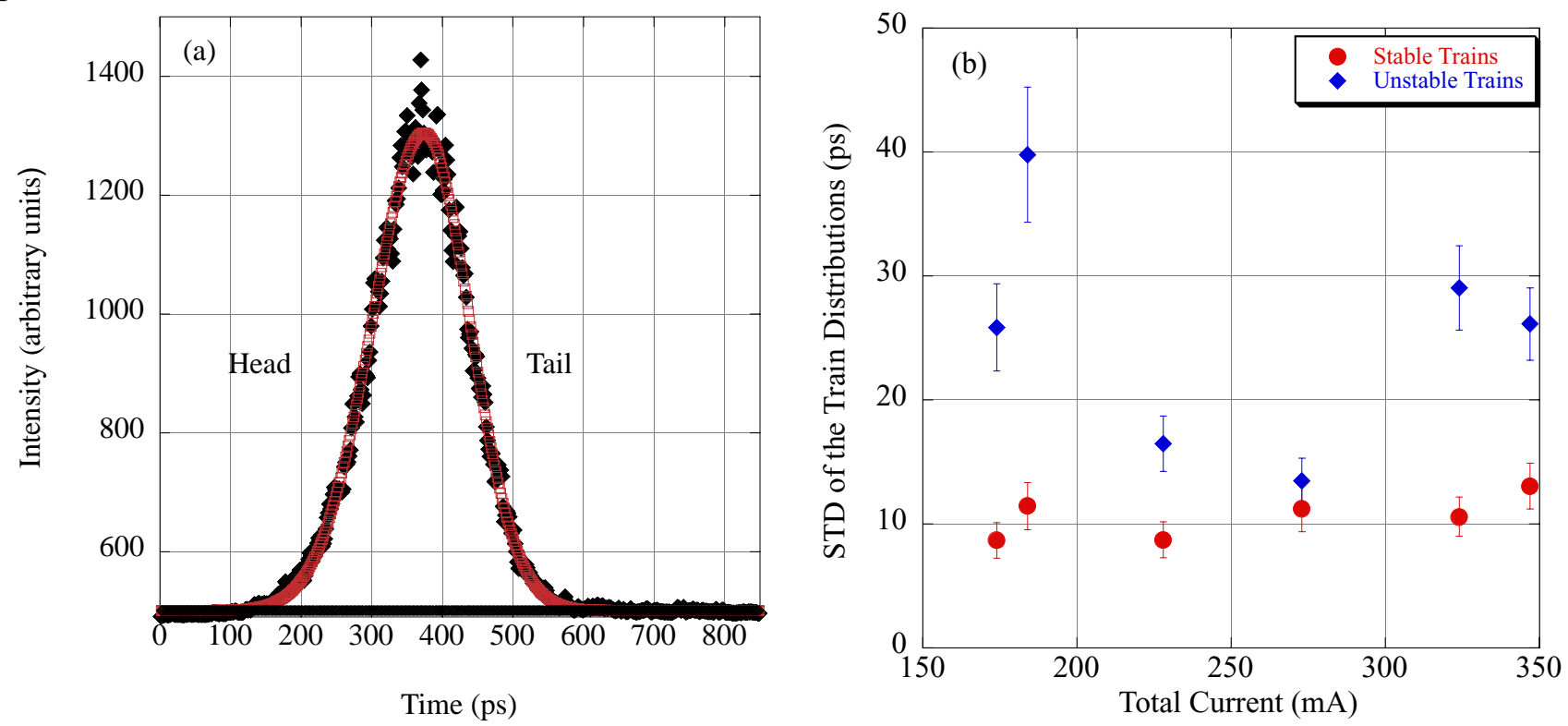

FIG. 26. (Color) (a) A typical longitudinal profile of a train fit to an asymmetric Gaussian function. (b) At each beam current, the standard deviation train oscillation was determined with stable and unstable trains.

Several comments can be made about these results. (i) The standard deviation of the train's distribution's centroid time position is consistently around $10 \mathrm{ps}$ when the trains are stable. During unstable operation, the standard deviation increases by up to a factor of 4 depending on the amount of current in CESR, which implies that bunches may have oscillation amplitudes up to 40 ps. (ii) 
Figures 27(a)-27(c) graph the measured centroid under three different conditions, a stable beam, an unstable beam at low current, and an unstable beam at high current. For the unstable low current case [Fig. 27(b)], all the trains correlate in phase with a period three train oscillation, but they have slightly different amplitudes. For the unstable high current case [Fig. 27(c)], there appear period 1 and 1.25 oscillations suggesting the presence of more than a single unstable mode of oscillation.
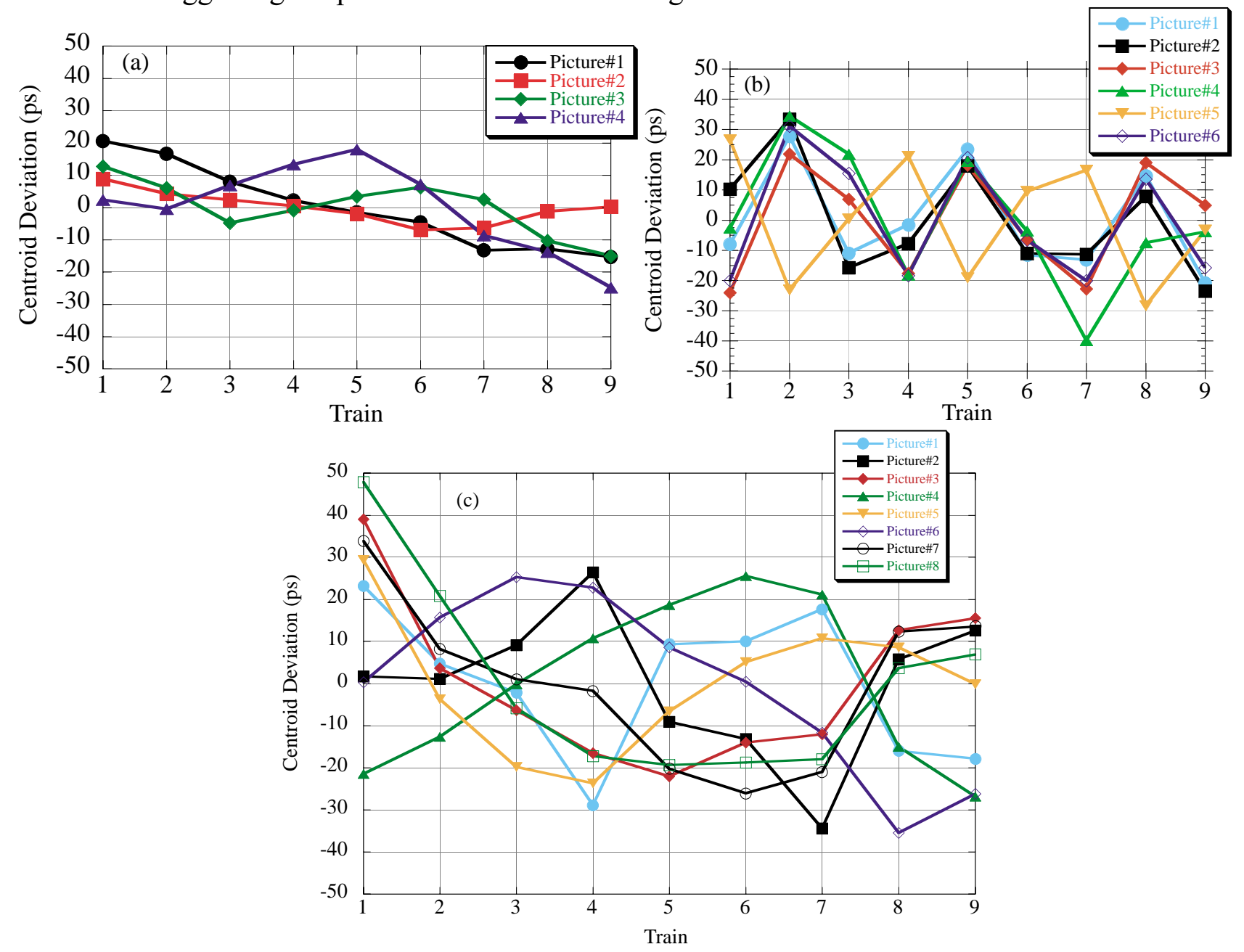

FIG. 27. (Color) The train centroid deviation for (a) four images of nine stable trains at $184 \mathrm{~mA}$ total current, (b) six images of unstable trains at $184 \mathrm{~mA}$ total current, and (c) eight images of unstable trains at $324 \mathrm{~mA}$ total current.

(iii) When the instability is present, the average temporal spread for each train is longer. Figure 28 is the longitudinal train length with and without the instability present. Again the spread in the distribution has two components, one due to the bunch length and the other proportional to the dipole oscillation amplitude. If the entire increase in standard deviation is due to an oscillation amplitude $\hat{z}$,

$$
\frac{1}{2} \hat{\mathrm{z}}^{2} \cong \sigma_{\text {unstable train }}^{2}-\sigma_{\text {stable train }}^{2},
$$

which gives a $8 \mathrm{~mm}$ peak amplitude averaged over the measurements shown in Fig. 28. In both cases, the train temporal spread grows with current, but at each current the train's temporal spread is larger with the instability present. (iv) Time oscillations have been measured for both bunches and trains. 
Since train oscillations have been measured, the long-range wakefields have sufficient fields at subsequent trains to produce bunch-train instabilities. (v) A comparison between bunch lengthening as a function of current, with a single bunch, or trains of bunches, is made. The growth rate with a single bunch present in CESR is $0.10 \mathrm{~mm} / \mathrm{mA}$, and the growth rate with multiple bunches is 0.21 $\mathrm{mm} / \mathrm{mA}$ [8]. The growth rate is almost a factor of 2 larger for trains than bunches. This points out that the interbunch wakefields give a large contribution to bunch lengthening.

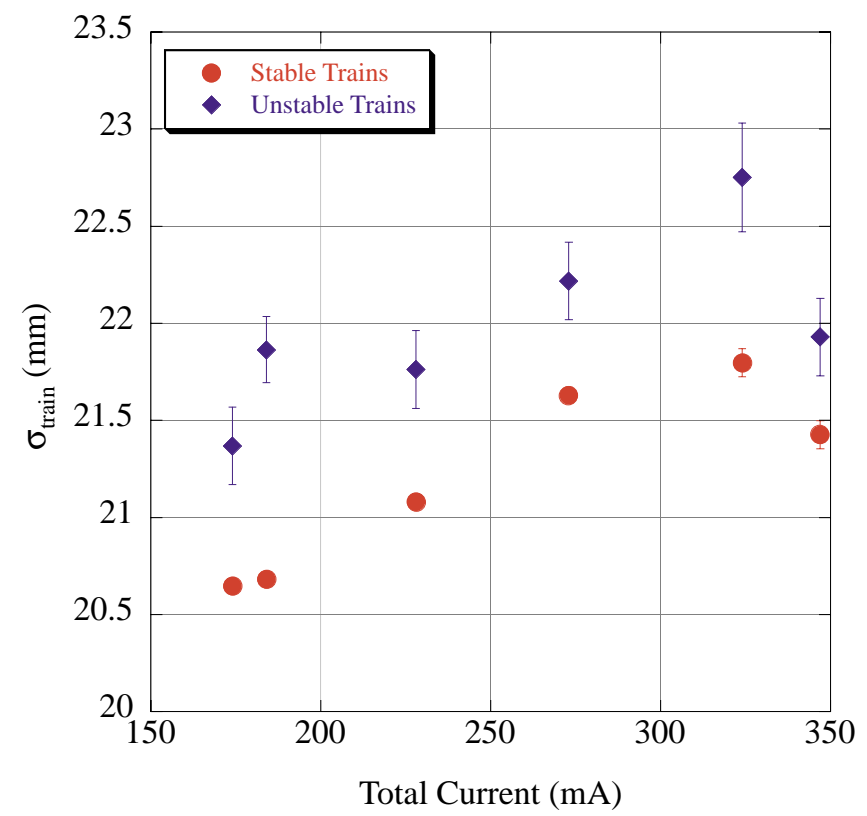

FIG. 28. (Color) The temporal spread of the trains with and without the instability present.

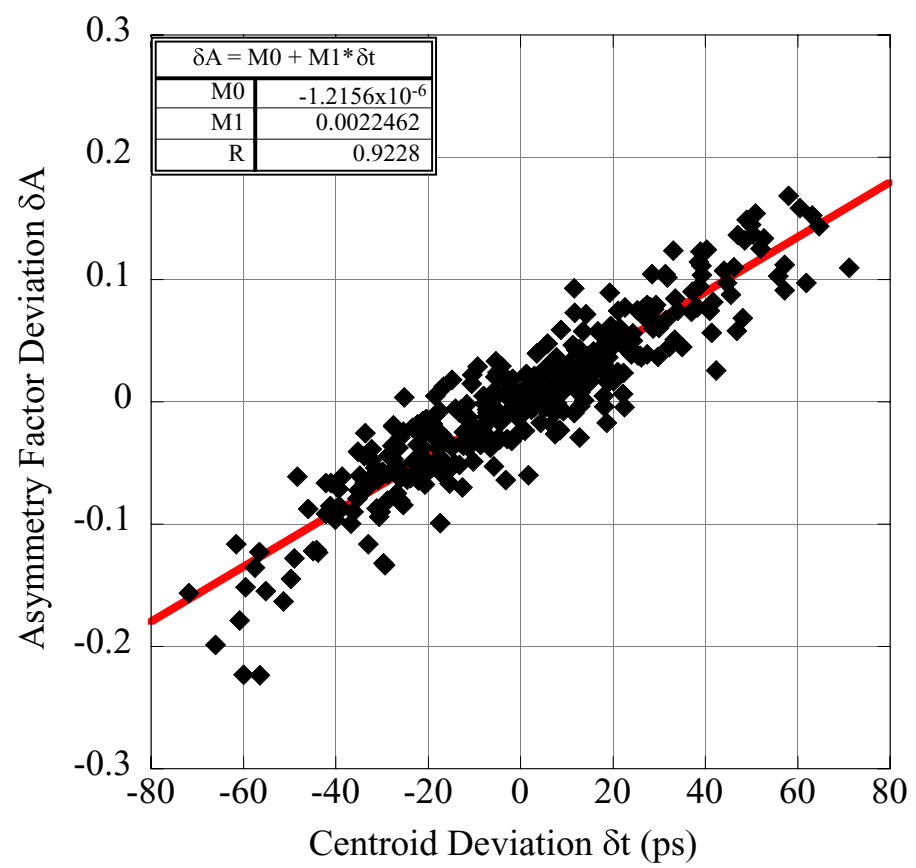

FIG. 29. (Color) The change in the asymmetry factor as a function of centroid deviation when the trains are unstable. 
The centroid deviation and asymmetry factor are highly correlated when the instabilities are present (Figs. 29). This suggests the following: (i) If the train arrives early, the train's asymmetry factor is negative (longer tail). It was pointed out in the previous section that a longer tail signifies bunches arriving early throughout the trains (an early arriving accordion mode). (ii) A late arriving train refers to bunches arriving later throughout the train (a late arriving accordion mode). Figure 30 is a pictorial description of the dynamics in the train.

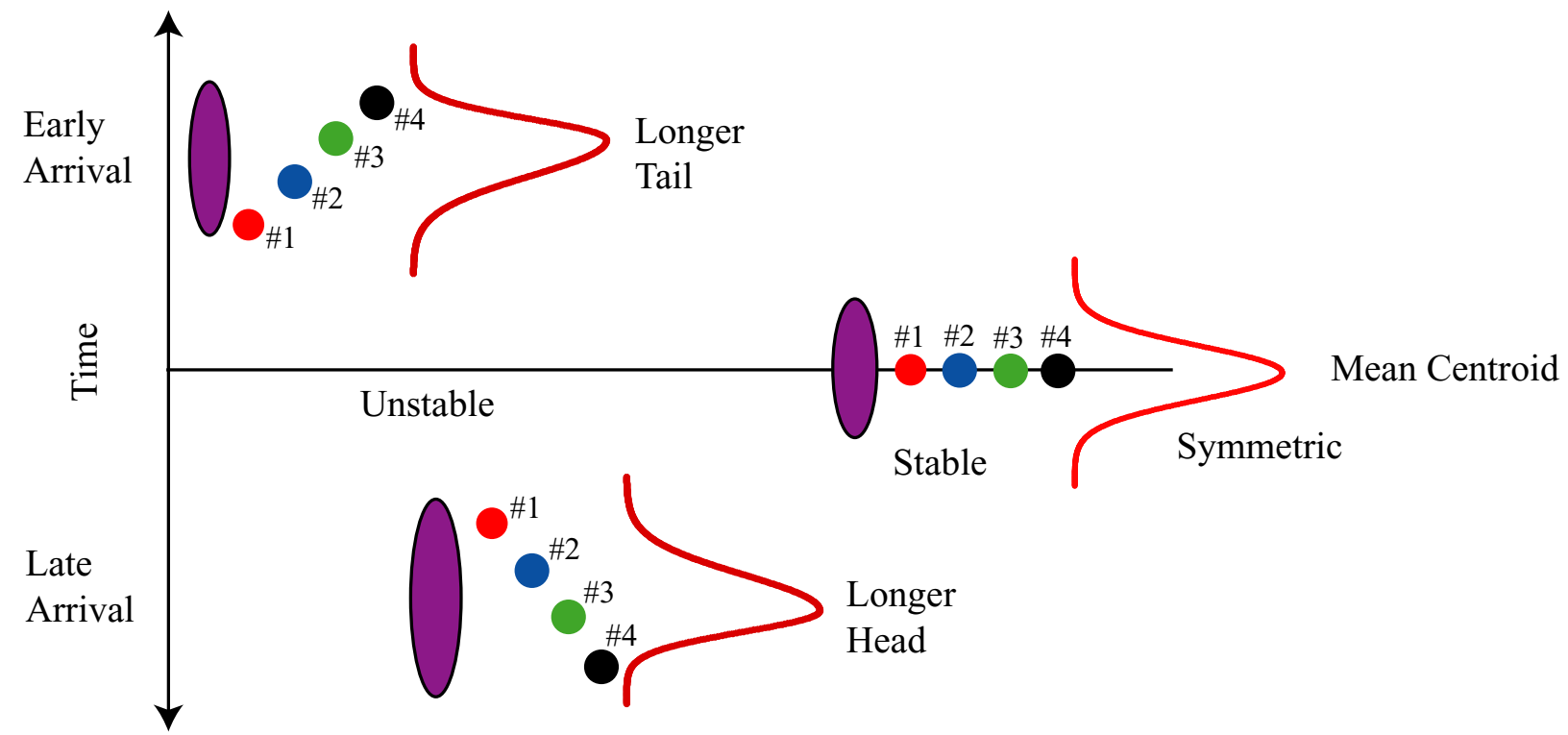

FIG. 30. (Color) The longitudinal dynamics with multiple trains present in CESR.

\section{Observations of colliding beams}

The primary mirror located in the CESR vacuum chamber extracts synchrotron radiation for both electrons and positrons. Therefore, it is possible to view both beams with the streak camera simultaneously. Since the primary mirror is located diametrically opposite the Cornell detector (CLEO), the pair of adjacent trains observed by the streak camera is the pair of trains that collides in CLEO. Therefore, longitudinal time oscillations of colliding beams can be measured with the streak camera.

A slight modification of the streak camera input optics was necessary to observe both bunches. This was accomplished by transporting the electron synchrotron radiation to the streak camera with an optical telescope. The positron synchrotron radiation was transported with the conventional optics.

The colliding beam experiment was performed during high-energy physics collisions when nine trains of five bunches of both electrons and positrons were present in CESR. Streak camera images were taken when the bunches were unstable and when they were stable. Typical representative images with stable (Fig. 31) and unstable (Fig. 32) motion with beams in collisions are presented.

Ideally, for stable collisions at the center of the CLEO detector, the time separation between bunches in the trains must remain constant. The goal of this experiment is to determine the time 
separation stability for colliding beams. The two different time oscillations, presented in the previous sections, measured for bunch collisions, are (i) train-to-train oscillations and (ii) bunch-to-bunch oscillations. Both oscillations require the following analysis of the longitudinal profiles.

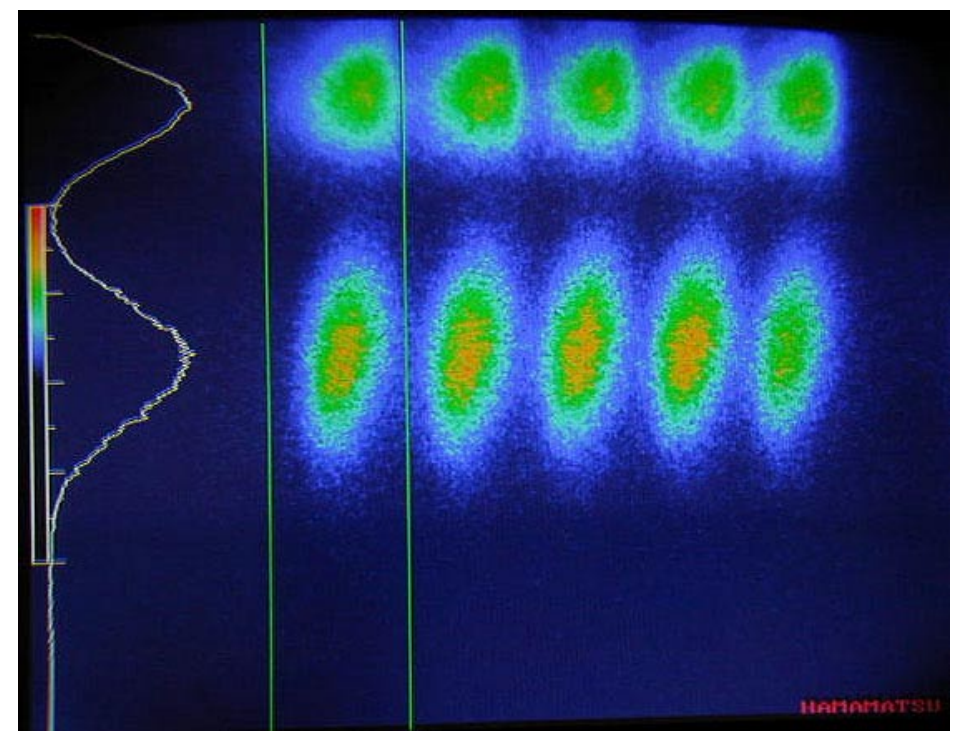

FIG. 31. (Color) A typical streak camera image of stable colliding beams in CESR. Bunch one in the train is to the left-hand side and bunch five is to the right-hand side on the horizontal axis. The train on the top is positrons, and on the bottom are electrons. The longitudinal profile is on the left-hand side of the figure. Under stable conditions, the bunches line up vertically.

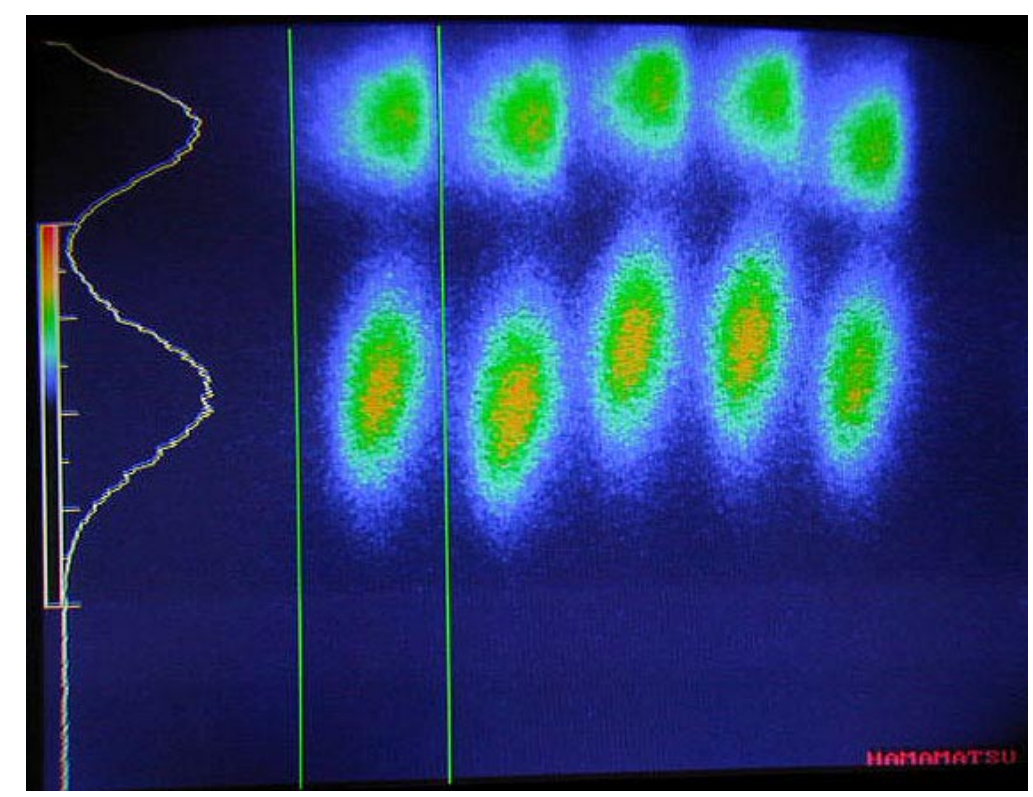

FIG. 32. (Color) A typical streak camera images of unstable colliding beams in CESR. The bunches (same arrangements as Fig. 31) in a train do not line up vertically.

For each image, the longitudinal profile of each colliding bunch (both electron and positron) is saved. Each profile is fit to a function that consists of two asymmetric Gaussian functions, one for each colliding beam. An example of a single profile fit to the two asymmetric Gaussian functions is 
displayed in Fig. 33. The mean position for each function determines the time separation between bunches.

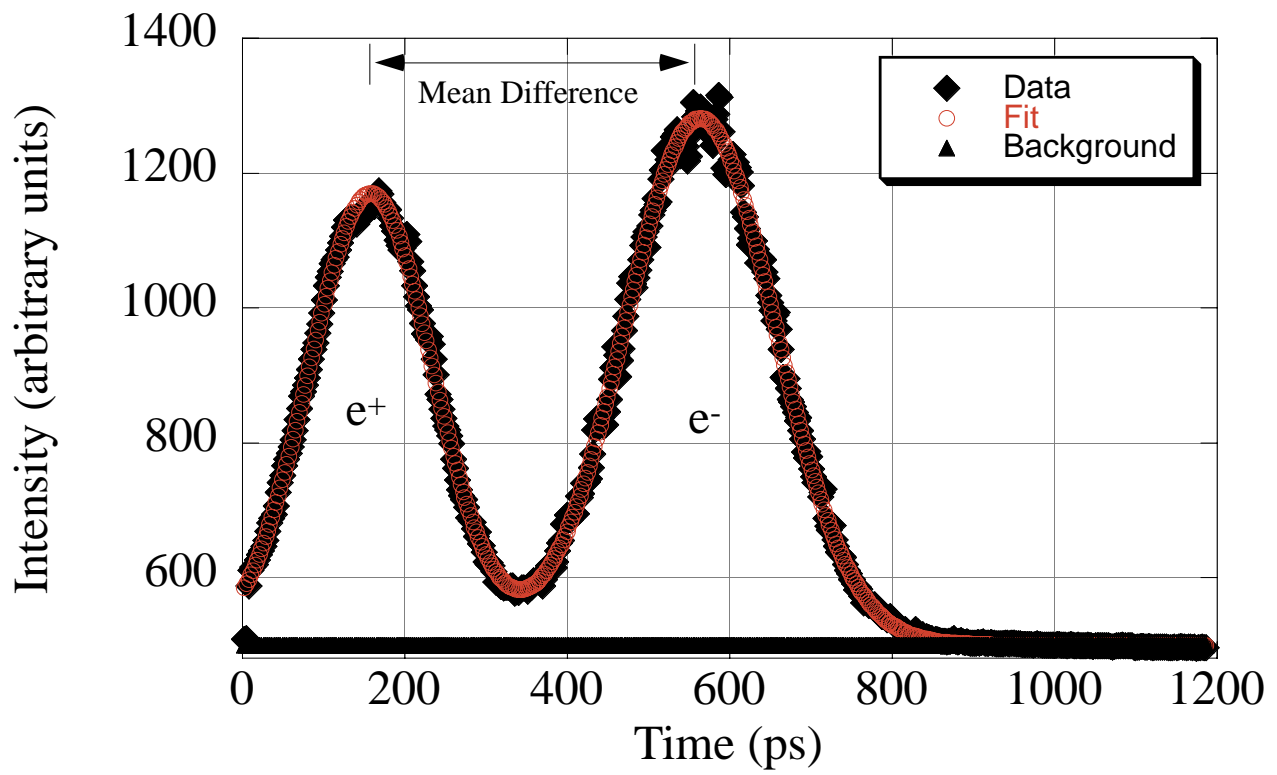

FIG. 33. (Color) A single profile of the electron and positron bunches which collide at the interaction point. The longitudinal distributions are fit and the distance between the distributions is computed.
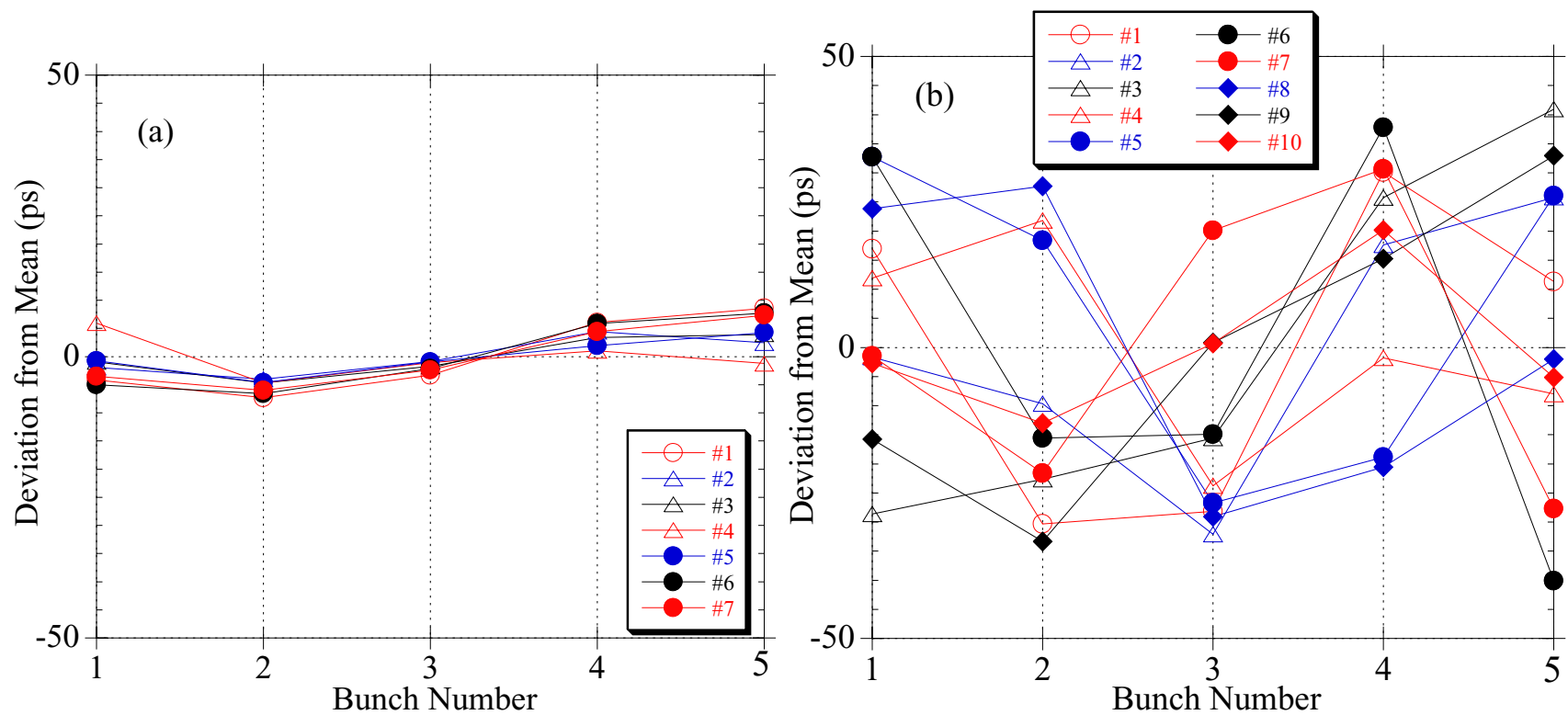

FIG. 34. (Color) The residual from the mean location for the bunches in the train when the bunches are (a) stable and (b) unstable.

For train-to-train time oscillations, the oscillations are determined by computing the train centroid for each image, for both electrons and positrons. The difference of train centroids for electrons and positrons is the time separation for each train. The mean time separation changed by $6.6 \mathrm{ps}$ from stable to unstable collisions.

Bunch-to-bunch time oscillations are characterized by the standard deviation of the mean centroid for each train during both stable and unstable collisions. The deviation from the mean position when 
collisions are stable and unstable is displayed in Fig. 34 for several streak camera images. The difference in the mean standard deviation between stable and unstable collisions is 29.6 ps.

The luminosity degradation due to the longitudinal dipole-coupled bunch instability for head-on collisions is estimated from Eq. (3). The time oscillation, from the bunch and train "hourglass" contributions, is $30 \mathrm{ps}$ or $9 \mathrm{~mm}$. This time oscillation changes the location of collision, and therefore increases the effective vertical betatron function $\beta_{\mathrm{v}}^{*}$. The reduction in luminosity from geometrical effects due only to the instability is given by the ratio of suppression factors

$$
\frac{L_{u}}{L_{s}}=\frac{s_{f}\left(\sigma_{z}\right)_{u}}{s_{f}\left(\sigma_{z}\right)_{s}}=\frac{0.763}{0.814}=0.94
$$

The luminosity was measured by the CLEO detector during this experiment and is displayed in Fig. 35. This reduction in luminosity measured by CLEO, approximately $24 \%$, is substantially larger than the $6 \%$ estimated luminosity reduction from bunch and train time oscillations. A more detailed model including crossing angles and coupling from solenoid compensation is required to calculate an accurate estimate of luminosity reduction from the longitudinal oscillations.

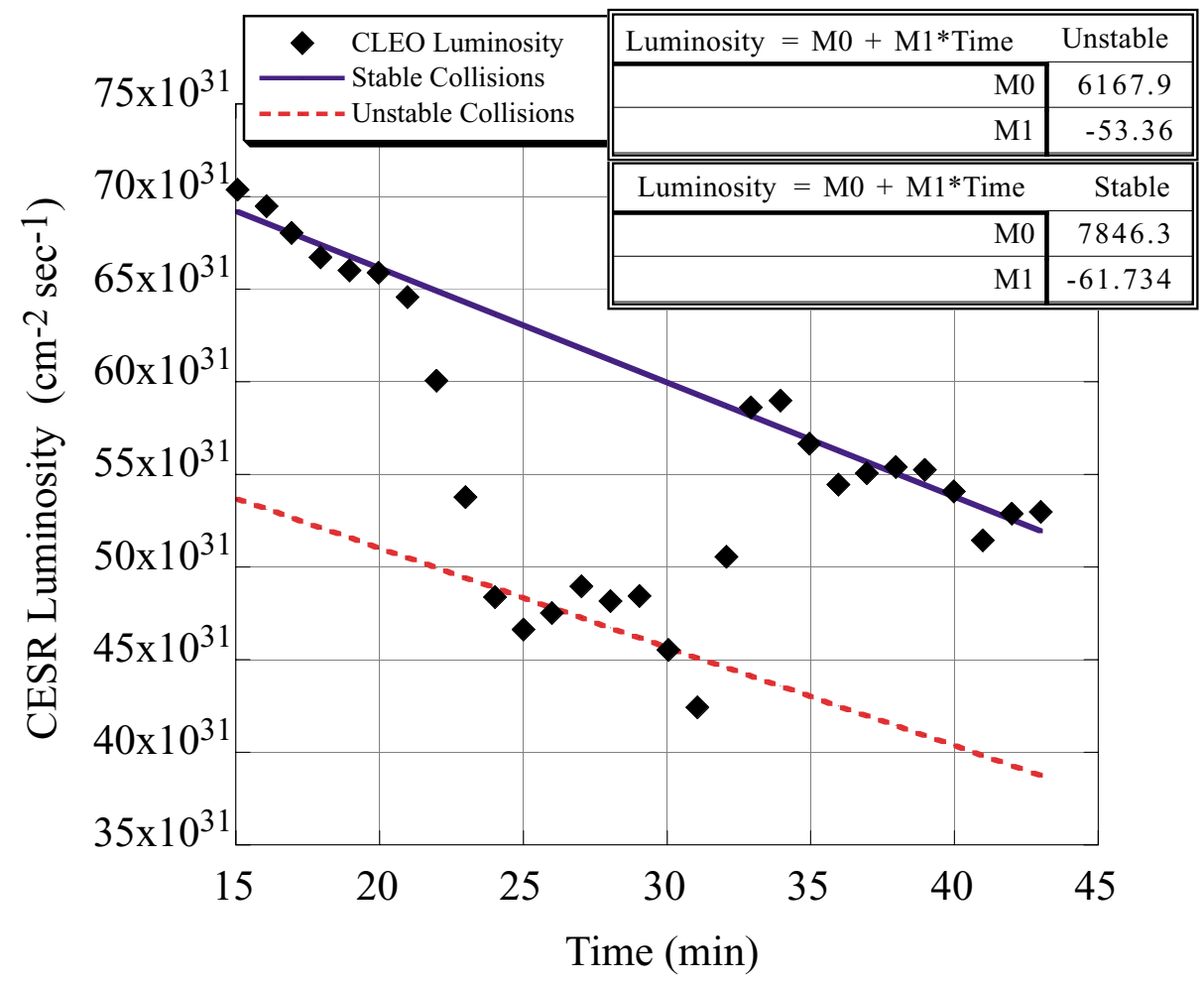

FIG. 35. (Color) A plot of the CLEO luminosity as a function of time. The dip in the luminosity occurred when the longitudinal feedback system was turned off and the beams were unstable. 


\section{Conclusions}

In this paper, we report the results of detailed measurements of the longitudinal dipole-coupled bunch instability at CESR. The measurements were made using a dual-sweep synchroscan streak camera. The experiments have allowed us to understand the longitudinal dynamics of the instability during high-energy physics collisions. The instability is damped by a strong feedback system that reduces its effect on luminosity and allows an increase in current threshold.

Measurements of single train, multiple trains and bunch collisions were performed. For a single train of bunches, we have concluded that: (i) above the instability threshold, the longitudinal feedback system stabilizes the temporal oscillations to approximately 5 ps. Without feedback, the temporal oscillations have peak amplitudes of approximately 100 ps. These oscillations, called bunch oscillations, degrade the high-energy physics luminosity. (ii) The oscillation modes within a train change as a function of current. Just above the instability threshold, an "accordion" mode of oscillation has been observed. The accordion mode has all the bunches in a train moving either together or apart in unison. At higher currents more complicated modes of oscillation are present. (iii) The instability is less severe with the superconducting rf cavities. Previously, the instability would produce shape oscillations at high current and this would lead to beam loss [3]. The superconducting cavities have made a significant difference in the characteristics of the instability. (iv) These bunch oscillations change the symmetry of the bunch distribution, which gives evidence for "breathing" in the bunch distribution. The asymmetry of the bunch distribution changes depending on its arrival time from a synchronous bunch. Figure 3 displays the change in the bunch's asymmetry as it oscillates in its potential well.

For multiple trains bunches in CESR, we have concluded that (i) trains have temporal oscillations, much like bunch oscillations, due to the longitudinal dipole-coupled bunch instability. These modes of oscillation can be represented as train modes. (ii) Train-to-train centroid motion has been measured. The maximum train centroid oscillation is approximately $50 \mathrm{ps}$ with and $10 \mathrm{ps}$ without the instability. (iii) Two modes of oscillation were measured, a period 3 and a combination of period 1 and 1.25 oscillation mode. (iv) The average longitudinal distribution of the bunches in a train grows faster than the single bunch length as a function of current. This is due to the interbunch wakefields. (v) At large oscillation amplitudes, the train distribution is highly asymmetric and results in large accordion oscillations of the bunches in a train. A pictorial description of the time oscillation of trains and bunches is displayed in Fig. 30.

Beam dynamics of colliding bunches were also measured. We can conclude from these measurements that (i) the instability's degradation of the luminosity is due to train and bunch oscillations. These oscillations reduce the luminosity by more than $20 \%$. (ii) A better model of CESR's interaction region optical characteristics is required to accurately estimate the luminosity reduction. 
The operation of the dual-sweep synchroscan streak camera is understood. The camera has provided detailed measurements of the longitudinal dynamics of the longitudinal dipole-coupled bunch instability that could not be measured by a normal streak camera. The camera has proved to be a valuable diagnostic tool for characterizing beam oscillations and longitudinal beam dynamics for the Cornell Electron-Positron Storage Ring.

\section{Acknowledgments}

The authors would like to thank the operation staff at the Laboratory of Nuclear Studies at Cornell University. We would also like to thank Robert Meller, David Rice, Robert Siemann, and Sasha Temnykh for useful discussions about the dynamics observed.

\section{References}

[1] Sikora, J., et al., "Longitudinal Feedback at CESR". Cornell University, Colliding Beam Note 99-20, 1999. Proceedings of the 1999 Particle Accelerator Conference.

Billing, M., et al., "Performance of the Beam Stabilizing Feedback Systems at CESR". To be published in the 2001 Particle Accelerator Conference proceedings.

[2] Belomestnykh, S., et al., "Superconducting RF System for the CESR Luminosity Upgrade: Design, Status, Plans". SRF 960529-02.

[3] Holtzapple, R.L., et al., "Multiple Bunch Longitudinal Dynamics Measurements of the Cornell Electron-Positron Storage Ring ". Phys. Rev. ST Accel Beams 4, 014401, 2001.

[4] Sands, M., "The Physics of Electron Storage Rings: An Introduction", SLAC, SLAC-R-0121, Nov 1970. 172pp.

[5] Billing, M., "Observation of a Longitudinal Coupled Bunch Instability with Trains of Bunches in CESR," CLNS 98/1564.

[6] Chao, A.W., "Physics of Collective Beam Instabilities in High-Energy Accelerators", New York, USA:Wiley (1993) 371p.

[7] Milton, S., "Calculation of How the Ratio $\beta^{*} / \sigma_{\text {bunch length Affects the Maximum Luminosity }}$ Obtainable: The 'Hourglass Effect'.", Cornell University, Colliding Beam Note 89-1, 1989.

[8] Holtzapple, R.L. and Rice, D., "Single Bunch Longitudinal Measurements of the Cornell ElectronPositron Storage Ring with the Superconducting RF Cavities." Cornell University, Colliding Beam Note $01-1,2001$.

[9] Billing, M., "Modes of Oscillation of Trains of Bunches." Cornell University, Colliding Beam Note 99-2, 1999. 